\title{
Estimation of ionospheric delay of NavIC/IRNSS signals using the Taylor Series Expansion
}

\author{
Mehul V. Desai* and Shweta N. Shah \\ Sardar Vallabhbhai National Institute of Technology (SVNIT), Ichchhanath Surat-Dumas Road, Keval Chowk, Surat, 395007 Gujarat, India
}

Received 2 September 2018 / Accepted 28 May 2019

\begin{abstract}
The delay in Navigation with Indian Constellation (NavIC)/Indian Regional Navigation Satellite System (IRNSS) signals due to the ionosphere are decisive because it leads to significant changes in the positional accuracy of the system. In this paper, we try to estimate the ionospheric time delay (ionodelay) precisely using the local Taylor Series Expansion (TSE) algorithm for a single frequency NavIC/IRNSS system. The performance of the local TSE algorithm is examined by considering two cases. In case I, the TSE was validated under the influence of an intense geomagnetic storm (8 September 2017) by considering NavIC/IRNSS data from the Indian equatorial and Equatorial Ionization Anomaly (EIA) regions. In case II, based on the quiet and disturbed days data, the local TSE model was examined at different locations in the local area $(<10 \mathrm{~km})$ using two NavIC/IRNSS receivers (i.e. reference and rover). The results of ionodelay and positional accuracy (Three Dimensional Distance Root Mean Square [3DRMS], Circular Error Probability [CEP], and Spherical Error Probability [SEP]) of NavIC/IRNSS for both the cases indicates that the single frequency local TSE algorithm performs the same as the reference dual frequency model, where as the global eight coefficient Klobuchar and the regional Grid Ionospheric Vertical Error (GIVE) model behaves differently. Therefore, the single-frequency TSE model improves the performance of the NavIC/IRNSS receiver in the local area, and the mathematical coefficient computation and additional frequency hardware cost have been reduced, with the acceptance of a maximum $0.8 \mathrm{~m}$ of errors.
\end{abstract}

Keywords: Navigation with Indian Constellation (NavIC) / Indian Regional Navigation Satellite System (IRNSS) / coefficient based model / regional model / Taylor Series Expansion (TSE) / ionospheric delay / positional accuracy

\section{Introduction}

The Navigation with Indian Constellation (NavIC)/Indian Regional Navigation Satellite System (NavIC/IRNSS), developed by the Indian Space Research Organization (ISRO), is in the final stages of development into a complete, autonomous regional navigation system (Desai et al., 2016; Desai \& Shah, 2017, 2018a, 2019). The NavIC/IRNSS will provide precise positional accuracy of the user anywhere and anytime in the Indian region using the signal in $\mathrm{L}_{5}$-band (1164.45$1188.45 \mathrm{MHz})$ and S-band (2483.5-2500 MHz) with a carrier frequency of $1176.45 \mathrm{MHz}\left(f_{1}\right)$ and $2492.08 \mathrm{MHz}\left(f_{2}\right)$ respectively (https://www.isro.gov.in/sites/default/files/irnss_icd_ version1.1-2017.pdf). Currently, seven NavIC/IRNSS satellites $1 \mathrm{~B}, 1 \mathrm{C}, 1 \mathrm{D}, 1 \mathrm{E}, 1 \mathrm{~F}, 1 \mathrm{G}$, and $1 \mathrm{I}$ are active in the orbit (http:// Www.isro.gov.in/Applications/SatelliteNavigationProgram).

\footnotetext{
*Corresponding author: mvd. svnit@gmail.com
}

The positioning accuracy of the satellite based navigation system is affected by satellite geometry, signal reception delays due to ionosphere and troposphere, multipath, Doppler effect due to relative motion, clock drift and receiver noise. Desai et al. (2016), the authors of this paper analyzed the effects of satellites geometry in terms of various DOPs parameters for NavIC/IRNSS and enhanced NavIC/IRNSS + GPS systems. Of all the effects discussed above, the influence of the ionosphere dominates because of the small delay of the signal affects the user (receiver) position considerably. Also, at the equator and lower latitudes of India, the presence of a wide range of spatial and temporal electronic gradient, NavIC/IRNSS signals get more pretentious by the ionosphere (Bhushana Rao, 2008; Sunehra et al., 2010). Therefore, a suitable ionospheric delay mitigation model is essential.

The delay or distortion of the navigation signal is proportional to the total electron content of the ionosphere in that region (Raveendran et al., 2017). To estimate total electron content, there are two types of models available (I) global (II) 
regional. The coefficient based single frequency Klobuchar (Klobuchar, 1987), refinement of global Klobuchar ionospheric coefficients (Wang et al., 2016, 2019), the Taiwan Ionospheric Model (TWIM) (Macalalad et al., 2013), International Reference Ionosphere (IRI), and Global Ionospheric Map (GIM) are examples of Global model, Where, the various grid based models like Minimum Mean Square Error (Ratnam \& Sarma, 2006), Krigin (Venkata Ratnam et al., 2011), Inverse Distance Weighted (IDW) with the Klobuchar model (Sarma et al., 2006), planar fit model (Sarma et al., 2009), Spherical Harmonic Function (SHF) model (Ratnam \& Sarma, 2012), modified Klobuchar model for two shell model (Shukla et al., 2013), Anisotrophic IDW with jackknife (Srinivas et al., 2016), are examples of the regional model that have been applied in the low latitude Indian region.

Desai \& Shah (2015), the authors of this paper have conducted a detailed survey related to suitability of different ionodelay models described above for the NavIC/IRNSS system. The author also observed that for the NavIC/IRNSS system the regional Grid Ionospheric Vertical Error (GIVE) model performed well compared to a global Klobuchar model (Desai \& Shah, 2017, 2018a). In the course of seasonal activities, magnetic storms and daily activities, total electron content can be accurately estimated by the dual-frequency method, but the use of the regional model is difficult, therefore, some local models are applied by Fujita, Seigo et al. (Fujita et al., 2009, 2010) to estimates ionodelay accurately over the Japan region. Similarly, in the low latitude Indian region also the large temporal and spatial ionospheric gradients are always present, hence to improve the positional accuracy of the NavIC/ IRNSS system the local model is needed to estimate ionodelay precisely.

The local Taylor Series Expansion (TSE) model is applied to GPS Aided GEO Augmented System (GAGAN) for the Indian region by Kumar et al. (2014). They observed that compared to the regional model, the local model performs better for quiet days than the disturbed days for all the 17 stations. But, the application of the TSE model on NavIC/IRNSS will be an open research which being incorporated by presenting less complex, local TSE in this paper, as the upcoming NavIC/IRNSS system faces high ionospheric effects due to the low latitude region. The order of TSE is changed in such a way that the local TSE model will work efficiently even in the intense geomagnetic days for NavIC/IRNSS System. Here, at the transmitter side, based on the vertical total electron content measured using dual frequency NavIC/IRNSS receiver the TSE coefficients are calculated. The users of nearby the NavIC/IRNSS receiver can use this TSE coefficient to estimate the ionodelay precisely.

In this paper, we considered two test setup, the TSE coefficient generation and ionodelay estimation using (I) single receiver (II) two different receivers. In case (I), i.e. using one NavIC/ IRNSS receiver, the TSE algorithm performance is examined during the intense $\left(D_{\mathrm{st}}=-124, K_{\mathrm{p}}=8, A_{\mathrm{p}}=106\right)$ geomagnetic storm beginning from 8 September 2017 using the one week of NavIC/IRNSS receiver data (3-9 September 2017) of the Indian equator (IIST Trivandrum) and four Equatorial Ionization Anomaly (EIA) (SVNIT Surat, IIT Bombay, CBIT Hyderabad, IIT Gandhinagar) location. In Desai \& Shah (2018b), we studied this intense geomagnetic storm and their impacts on NavIC/ IRNSS system with detailed. The single-frequency local TSE and regional GIVE model performances are correlated with the reference dual-frequency model for verification.

In case (II), we define $10 \mathrm{~km}$ region as a local region and based on the data (quiet and disturb days) availability of two receivers (i.e. reference and rover) the performance of the local TSE model is examined. Further optimization in the positional accuracy is achieved by applying, Hopfield model for the tropospheric delay and Iterative Least Square (ILS) algorithm for position estimation. The local TSE algorithm is verified by comparing the performance in terms of Three Dimensional Distance Root Mean Square (3DRMS), Circular Error Probability (CEP) and Spherical Error Probability (SEP) with the different single frequency (i.e. global Klobuchar and regional GIVE model) and reference dual frequency model. It is deduced from the analysis of both cases that single frequency TSE model performed nearly the same as the reference dual frequency model and better performed compared to global Klobuchar and regional GIVE model. Therefore, the TSE model does not only estimating ionodelay precisely in the local region but also reducing the cost of extra hardware frequency.

The structure of this paper is as follows. Detailed mathematical information on the proposed work and supporting calculations is presented in Section 2. Test setup and data collection procedures are explained in Section 3. Section 4 provides a simulation analysis of the NavIC/IRNSS system for ionodelay and position error calculations. Finally, conclusion and future work are presented.

\section{2 lonodelay estimation using the local TSE model}

The user's 3D position in the World Geodetic System (WGS-84) format is determined by finding the intersection point of the observed ranges from at least three satellites, but one more satellite range observation is required to resolve timing offset problem (Misra \& Enge, 2006; Desai et al., 2016). As the code measurements always suffer from various error sources, the range measured by different satellites is not true, but it is psuedorange and represented as a $\rho_{\mathrm{pr}}$ and given by (Misra \& Enge, 2006; Desai \& Shah, 2015, 2018a; Desai et al., 2016),

$$
\begin{aligned}
\rho_{\mathrm{pr}} & =c\left[\left(T^{u}+t^{u}\right)-\left(T^{s}-\delta^{s}\right)\right]+d_{\mathrm{pr}}+m p_{\mathrm{pr}}+n_{\mathrm{pr}} \\
& \left.=c\left(T^{u}-T^{s}\right)+c\left(t^{u}-\delta^{s}\right)\right]+d_{\mathrm{pr}}+m p_{\mathrm{pr}}+n_{\mathrm{pr}} \\
\rho_{\mathrm{pr}_{i}}^{k} & =R_{i}^{k}+c\left(\Delta_{t_{i}}\right)^{k}+d_{\mathrm{pr}_{i}}^{k}+m p_{\mathrm{pr}_{i}}^{k}+n_{\mathrm{pr}_{i}}^{k}
\end{aligned}
$$

where, $T^{u}$ and $T^{s}$ are the time instants when signal left from the satellites and signal reached at the satellites, respectively. Similarly, $t^{u}$ and $\delta^{s}$ are the clocks offset from system time for receiver and satellite respectively. The $c$ is the velocity of propagation, $\Delta_{t}$ is the total time offset between satellites and receiver. The $R_{i}^{k}$ is a true distance between the $i$ th satellite and $k$ th user (Desai et al., 2016). It can be calculated using,

$$
R_{i}^{k}=\sqrt{\left(x_{i}^{s}-x^{u}\right)^{2}+\left(y_{i}^{s}-y^{u}\right)^{2}+\left(z_{i}^{s}-z^{u}\right)^{2}}
$$


When four pseudoranges are observed, then $i$ ranges from 1 to 4. $\left(X^{s}, Y^{s}, Z^{s}\right)$ denotes 3D known geocentric coordinates of satellites and $\left(X^{u}, Y^{u}, Z^{u}\right)$ are unknown geocentric coordinates of the user which are to be computed (Sarma et al., 2010). Similarly, $d_{\mathrm{pr}_{i}}^{k}$ is the total atmospheric delay suffered by $i$ th satellites can be represented as,

$$
d_{\mathrm{pr}_{i}}^{k}=I_{\mathrm{pr}_{i}}^{k}+T_{\mathrm{pr}_{i}}^{k}
$$

where, $I_{\mathrm{pr}_{i}}^{k}$ code delay due to the ionosphere, which will be always positive in magnitude and $T_{\mathrm{pr}_{i}}^{k}$ is the code delay because of troposphere which is independent of frequency. The $m p_{\mathrm{pr}_{i}}^{k}$ and $n_{p r_{i}}^{k}$ shows the stochastic characteristics of psuedorange due to multipath delay and other measurement noise (Misra \& Enge, 2006; Shi et al., 2012; Desai et al., 2016). As the ionosphere is a dispersive medium, delays at different frequencies are different. The ionodelay $\left(I_{\mathrm{pr}_{j}}^{k}\right)$ for the NavIC/IRNSS satellites $i=1,2, \ldots, 7$ and receiver $k$ can be estimated to be proportional to the vertical total electron content (Desai \& Shah, 2018b),

$$
I_{\mathrm{pr}_{i}}^{k}=\text { Ionodelay }_{\mathrm{L}_{5} / \mathrm{S}}=\frac{40.3 \times 10^{16}}{f^{2}} \times \mathrm{VTEC}_{i} \times F_{i}(\mathrm{~m})
$$

where $\mathrm{VTEC}_{i}$ is the vertical total electron content, it is measured by considering ionosphere as single thin shell $(350 \mathrm{~km})$ using dual frequency model for satellites based navigation system (Misra \& Enge, 2006). In this paper, we estimated it by the single frequency local TSE model for NavIC/IRNSS system, whose functional equation is represented as,

$$
\begin{aligned}
\mathrm{VTEC}_{i}= & \sum_{n=0}^{2} \sum_{m=0}^{1} \beta_{n m}\left\{\left(\phi_{\mathrm{IPP}_{i}}\right)^{n} \times\left(\lambda_{\mathrm{IPP}_{i}}\right)^{m}\right\} \\
= & \beta_{00}+\beta_{01} \lambda_{\mathrm{IPP}_{i}}+\beta_{10} \phi_{\mathrm{IPP}_{i}}+\beta_{11} \phi_{\mathrm{IPP}_{i}} \lambda_{\mathrm{IPP}_{i}} \\
& +\beta_{20} \phi_{\mathrm{IPP}_{i}}^{2}+\beta_{21} \phi_{\mathrm{IPP}_{i}}^{2} \lambda_{\mathrm{IPP}_{i}}
\end{aligned}
$$

The constellation of NavIC/IRNSS satellite arranged in such a away that all satellites always visible within the Indian region (Zaminpardaz et al., 2017). Before the 1I NavIC/IRNSS satellite (launched on 12 April 2018, 4:04 am IST) only six NavIC/ IRNSS were satellites active in the orbit (Desai \& Shah, 2019). Hence, the order of conventional TSE is change for six visible NavIC/IRNSS satellites (i.e. maximum $n=2$ and $m=1$ ) in such a way that estimated total electron content value was nearly same as total electron content measured by dual frequency approach. (Fujita et al., 2009, 2010), incorporated the effect of the order variation of TSE which applied here and its mathematical description is explained in the next section.

\subsection{Calculation of TSE coefficients on reference receiver side}

The calculation of TSE coefficients can be described by the following steps:

1. Calculate Ionospheric Pierce Point (IPP) Latitude and Longitude for the reference $t$ receiver: Here, the ionosphere is approximated by the single thin shell, hence the TEC is calculated at the IPP point of the single thin shell at the Line of Sight (LOS) between user and satellite (refer Fig. 1) (Misra \& Enge,

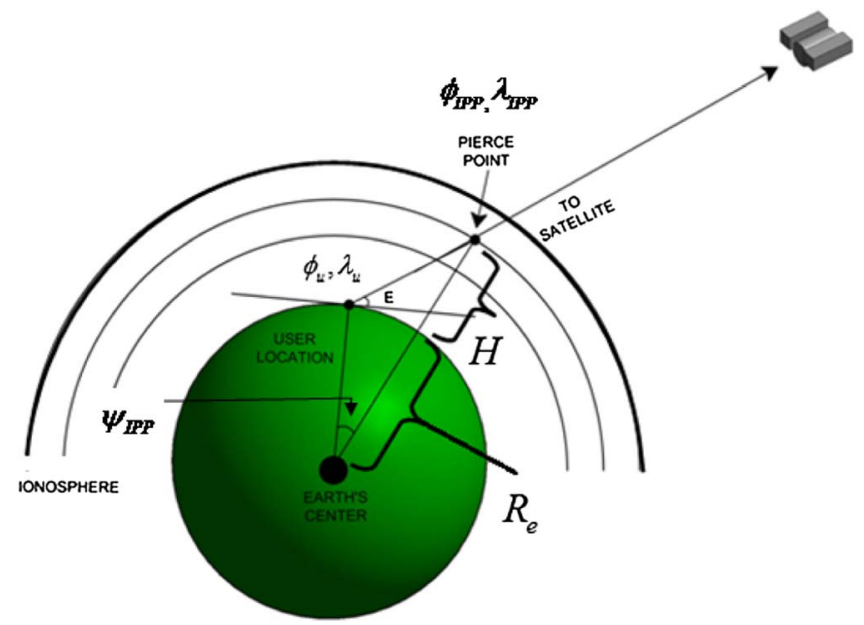

Fig. 1. Receiver satellite geometrical location.

2006; Kumar et al., 2014; Desai \& Shah, 2015, 2018a; Desai et al., 2016). The IPP latitude and longitude is calculated based on WGS84 geographic coordinate system as given by:

$$
\begin{aligned}
\phi_{\mathrm{IPP}_{i}}^{t}= & \sin ^{-1}\left\{\sin \left(\phi_{u}^{t}\right) \times \cos \left(\psi_{\mathrm{IPP}_{i}}^{t}\right)+\cos \left(\phi_{u}^{t}\right) \times \cos \left(A_{z_{i}}^{t}\right)\right. \\
& \left.\times \sin \left(\psi_{\mathrm{IPP}_{i}}^{t}\right)\right\},(\text { radian }), \\
\lambda_{\mathrm{IPP}_{i}}^{t}= & \lambda_{u}^{t}+\sin ^{-1}\left\{\frac{\sin \left(\psi_{\mathrm{IPP}_{i}}^{t}\right) \times \sin \left(A_{z_{i}}^{t}\right)}{\cos \left(\phi_{\mathrm{IPP}_{i}}^{t}\right)}\right\},(\text { radian })
\end{aligned}
$$

where, $\phi_{u}^{t}$ and $\lambda_{u}^{t}$ are latitude and longitude of the $t$ reference receiver, the $A_{z}^{t}$ denote azimuth from receiver to satellite, $\psi_{\operatorname{IPP}_{i}}^{t}$ is the angle between the line joining receiver to the center of the earth and the IPP to center of the earth and is calculated as:

$$
\begin{aligned}
\psi_{\mathrm{IPP}_{i}}^{t}= & \frac{\pi}{2}-E_{l_{i}}{ }^{t} \\
& -\sin ^{-1}\left\{\frac{R_{\mathrm{e}}}{R_{\mathrm{e}}+H} \times \cos \left(E_{l_{i}}^{t}\right)\right\},(\text { radian })
\end{aligned}
$$

where, $R_{\mathrm{e}}$ is the mean radius of the earth and is taken as $6378.1 \mathrm{~km}, E_{l}$ is the elevation angle in radian and $H$ is height for single thin ionosphere shell taken as $350 \mathrm{~km}$ above the earth's surface (Misra \& Enge, 2006; Kumar et al., 2014).

2. Calculation of observation matrix - The observational matrix $G_{\mathrm{k}}($ size $6 \times 6)$ is calculated based on active NavIC/ IRNSS satellites as,

$$
G_{\mathrm{k}_{i}}=\frac{40.3 \times 10^{16}}{f^{2}} \times F_{i} \times \sum_{n=0}^{2} \sum_{m=0}^{1}\left\{\left(\phi_{\operatorname{IPP}_{i}}{ }^{t}\right)^{n} \times\left(\lambda_{\operatorname{IPP}_{i}}{ }^{t}\right)^{m}\right\}
$$

3. Calculation of Mapping function - For the conversion of slant total electron content to vertical total electron content a mapping function is used. The elevation dependent mapping function is given by desai,

$$
F_{i}{ }^{t}=\sqrt{1-\left\{\frac{R_{\mathrm{e}} \times \cos \left(E_{l_{i}}{ }^{t}\right)}{R_{\mathrm{e}}+H}\right\}^{2}}
$$


4. Calculate the coefficients - The TSE coefficient matrix $X_{\mathrm{TSE}}=\left[\beta_{00}^{t} \beta_{01}^{t} \beta_{10}^{t} \beta_{11}^{t} \beta_{20}^{t} \beta_{21}^{t}\right]_{1 \times 6}^{T}$ can be calculated using Least Square (LS) estimation given by (Fujita et al., 2009),

$$
\begin{gathered}
\left(G_{k}^{T} G_{k}\right) X_{\mathrm{TSE}}=\left(G_{k}^{T} V^{t}\right) \\
\left(G_{k}^{T} G_{k}\right)^{-1}\left(G_{k}^{T} G_{k}\right) X_{\mathrm{TSE}}=\left(G_{k}^{T} G_{k}\right)^{-1}\left(G_{k}^{T} V^{t}\right) \\
X_{\mathrm{TSE}}=\left(G_{k}^{T} G_{k}\right)^{-1}\left(G_{k}^{T} V^{t}\right)
\end{gathered}
$$

where, $V^{t}$ matrix $\left(V^{t}=\left[\mathrm{VTEC}^{1 B} \mathrm{VTEC}^{1 C} \mathrm{VTEC}^{1 D} \mathrm{VTEC}^{1 E}\right.\right.$ $\left.\left.\mathrm{VTEC}^{1 F} \mathrm{VTEC}^{1 G}\right]_{1 \times 6}^{T}\right)$, consisting vertical total electron content value of active NavIC/IRNSS satellites measured by below dual frequency approach,

$$
\begin{aligned}
\mathrm{VTEC}= & \frac{1}{40.3 \times 10^{6} \times F} \times \frac{f_{1}^{2} \times f_{2}^{2}}{f_{1}^{2}-f_{2}^{2}} \\
& \times\left(P_{L_{5}}-P_{S}\right)\left(\text { electrons } / \mathrm{m}^{2}\right)
\end{aligned}
$$

where, $P_{L_{5}}$ and $P_{S}$ are pseudoranges measured in two frequencies $f_{1}$ (L $L_{5}$-band) and $f_{2}$ (S-band) of NavIC/IRNSS (Misra \& Enge, 2006; Desai et al., 2016). The reference receiver will broadcast these TSE coefficients every $5 \mathrm{~min}$ of the interval to provide an ionospheric correction for the rover receiver in the local $(<10 \mathrm{~km})$ region. Here, the duration is set to $5 \mathrm{~min}$, the same as the regional GIVE model, for proper comparison. The rover receiver will correct the ionospheric correction by following the mathematical steps.

\subsection{Applying TSE at rover receiver}

1. Calculate Latitude and Longitude at IPP: IPP latitude $\phi_{I P P_{i}}^{r}$ and $\lambda_{I P P_{i}}^{r}$ longitude at receiver is found out using the same formula mentioned in equation (6).

2. Calculation of vertical total electron content by using TSE coefficients: The vertical total electron content at the rover receiver is calculated using the transmitted coefficients $\left(X_{\mathrm{TSE}}\right)$ by the reference receiver using the simple formula,

$$
\mathrm{VTEC}^{r}=G_{r} \times X_{\mathrm{TSE}}
$$

which can be expanded as follows:

$$
\begin{aligned}
\mathrm{VTEC}^{r}= & \left\{\frac{40.3 \times 10^{16}}{f^{2}} \times \sum_{n=0}^{2} \sum_{m=0}^{1}\left\{\left(\phi_{\mathrm{IPP}_{i}}^{r}\right)^{n} \times\left(\lambda_{\mathrm{IPP}_{i}}^{r}\right)^{m}\right\}\right\} \\
& \times\left[X_{\mathrm{TSE}}^{1 B} \cdots X_{\mathrm{TSE}}^{1 G}\right]^{T}
\end{aligned}
$$

Finally, the ionodelay is calculated based on $\mathrm{VTEC}^{r}$ values at the rover receiver using formula defined in equation (4). The flow diagram TSE model graphically represented in Figure 2. At the single frequency rover receiver, true ionospheric delays (i.e. dual frequency delay) are not available, we have been estimated the delay using single frequency global Klobuchar, regional GIVE and local TSE model. Hence, measurement becomes stochastic (Shi et al., 2012) and there is always some error present called residual error and it is estimated by,

$$
\epsilon=I_{\mathrm{pr}_{i}}^{k}-\hat{I}_{\mathrm{pr}_{i}}^{k}
$$

where, $I_{\mathrm{pr}_{i}}^{k}$ is the ionodelay based on vertical total electron content value measured by dual frequency approach and $\hat{I}_{\mathrm{pr}_{i}}^{k}$
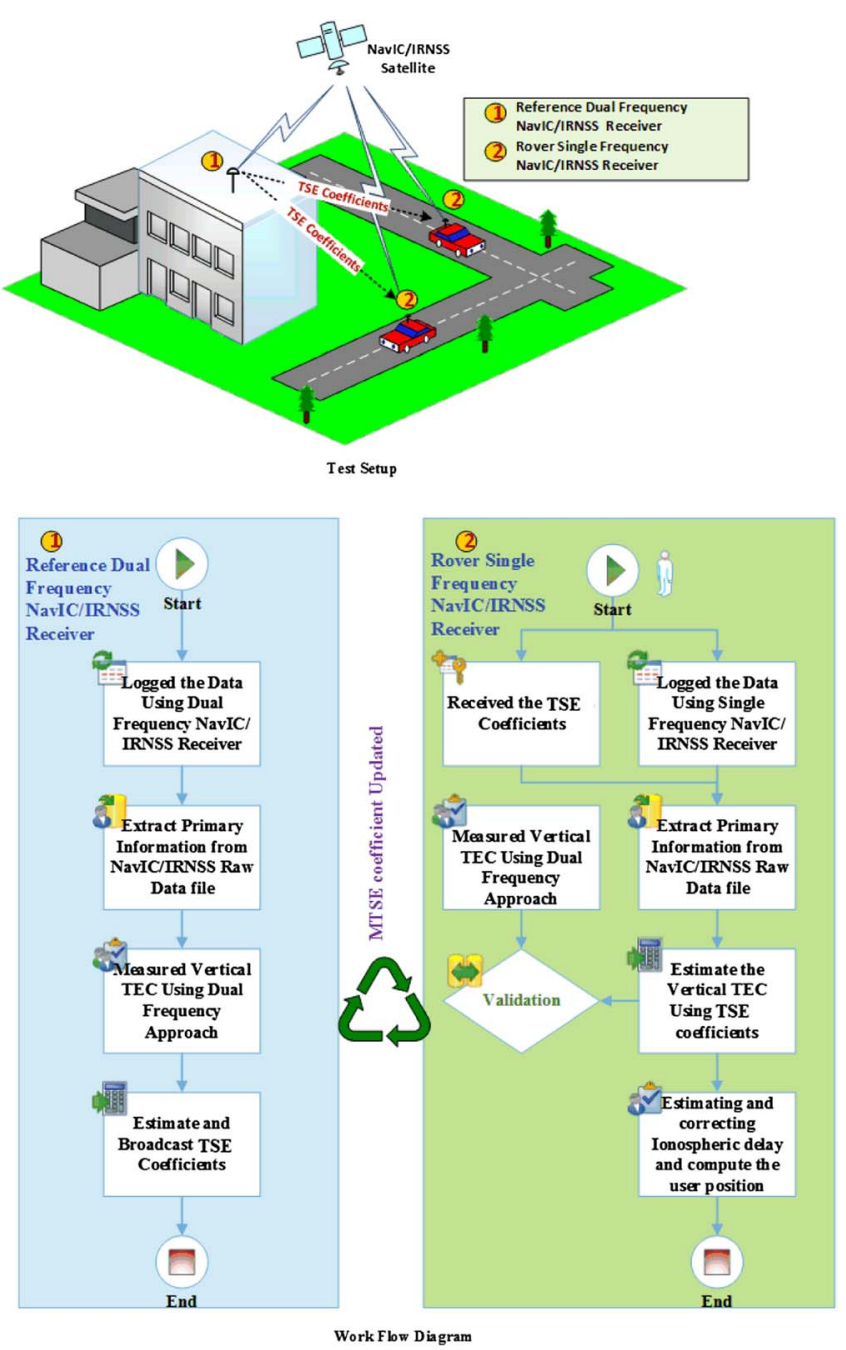

Fig. 2. Experimental setup and flow diagram.

is the ionodelay estimated by various single frequency models. The detail test setup and data collection for analysis of algorithms are included in the next section.

\section{Test setup and data collection}

The NavIC/IRNSS satellites data were collected using the Accord NavIC/IRNSS receiver which is provided by Space Application Center (SAC), Indian Space Research Organization (ISRO) Ahmedabad, India. The setup consists of antenna that sense the NavIC/IRNSS both band ( $\mathrm{L}_{5}$ and $\left.\mathrm{S}\right)$ and GPS $\mathrm{L}_{1}$-band signals. Jagiwala \& Shah (2018) reported that NaVIC/IRNSS $\mathrm{S}$-band is sensitive to intentional and unintentional error sources. Here, the further analysis of NavIC/IRNSS $\mathrm{L}_{5}$-band is explored for ionodelay and positional accuracy.

The experiment setup is depicted in Figure 2, the TSE coefficients are generated based on the vertical total electron content measured by the dual frequency NavIC/IRNSS reference receiver. The single frequency rover receivers attempt to estimate the vertical total electron content followed by ionodelay using the TSE coefficients produced by the reference dual 


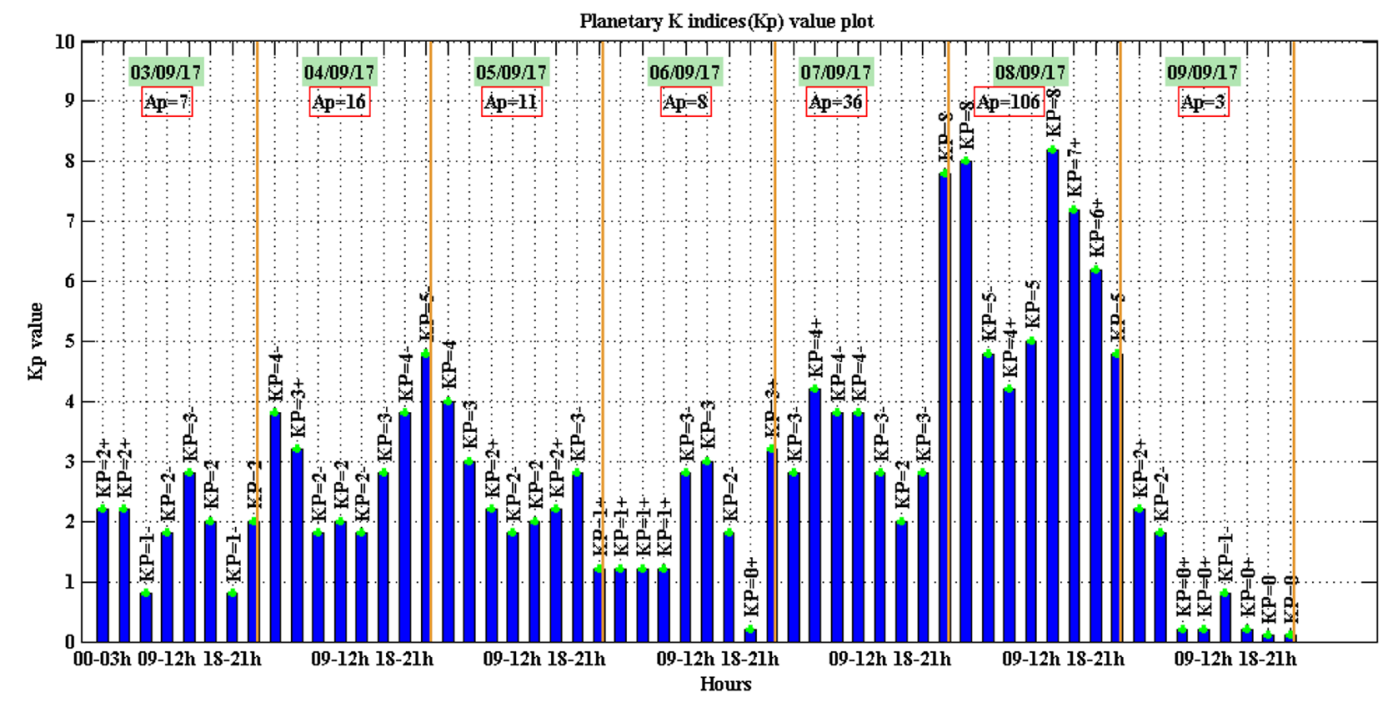

(a) $K_{p}$ Indices for 3 September to 9 September 2017

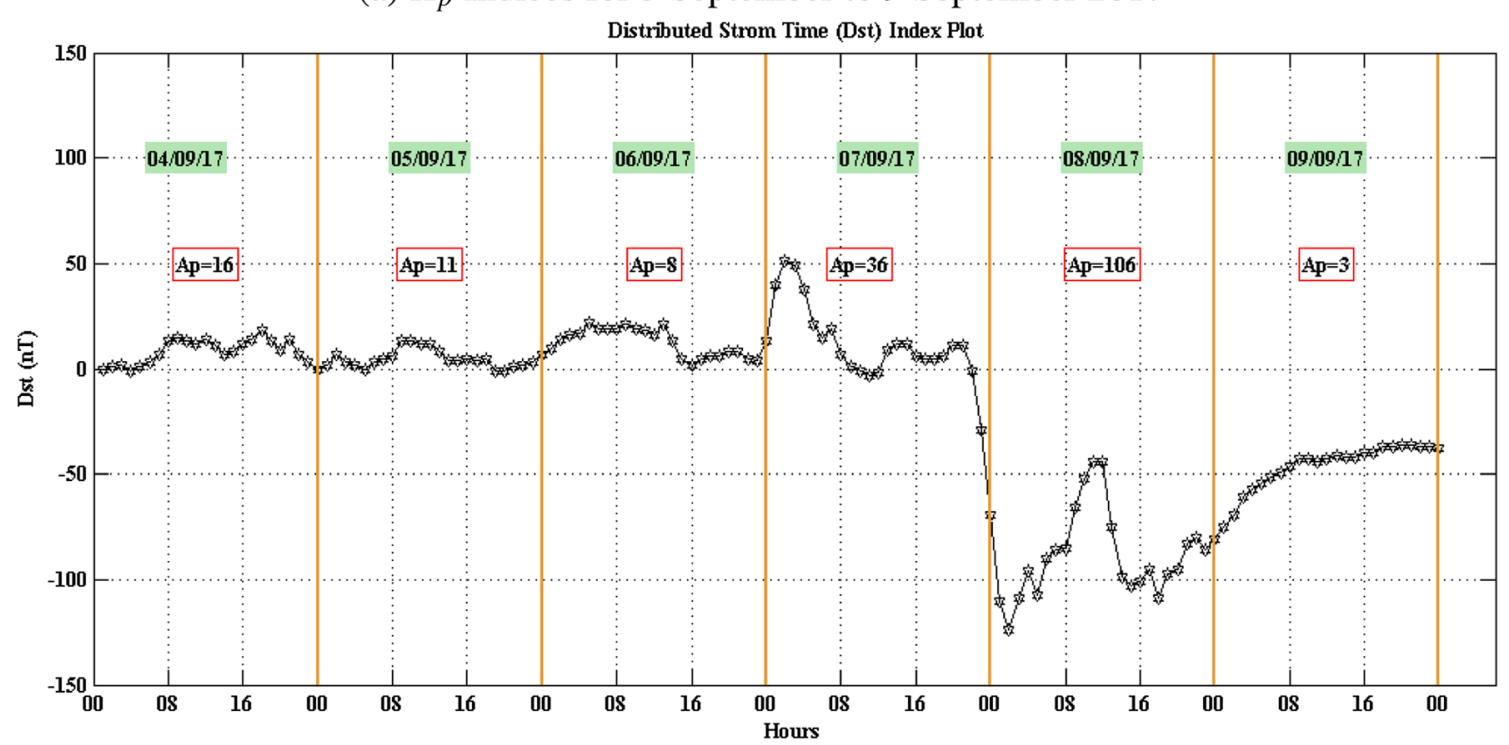

(b) $D_{s t}$ Index for 3 September to 9 September 2017

Fig. 3. Graphical representation of various geomagnetic parameters.

frequency receiver. This analysis takes into account two cases for algorithm validation are explained below.

\subsection{Case-I}

Here, the TSE algorithm analyzes by considering single NavIC/IRNSS receivers. This means that the TSE coefficient generation and the vertical TEC estimation (using the TSE coefficients) are done for static single NavIC/IRNSS receiver. In this case, the performance of the TSE algorithm is examined under the effect of an intense geomagnetic storm at various geographical location. The $D_{\text {st }}$ index from World Data Center (WDC) for geomagnetism, Kyoto (http://wdc.kugi.kyotou.ac.jp) and the $K_{\mathrm{p}}$ indices from OMNIWeb data explorer (https://omniweb.gsfc. nasa.gov) are used for the classification of quiet $\left(0<K_{\mathrm{p}}<1\right)$, disturbed/stormy $\left(K_{\mathrm{p}}>5\right)$ days.

According to the $D_{\mathrm{st}}$ and $K_{\mathrm{p}}$ index geomagnetic storm is classified as super $\left(D_{\mathrm{st}} \leq 250 \mathrm{nT}, K_{\mathrm{p}}=9\right)$, intense $\left(-100>D_{\mathrm{st}} \geq 250 \mathrm{nT}, K_{\mathrm{p}}=8\right)$, and medium $\left(D_{\mathrm{st}} \leq 100 \mathrm{nT}\right.$, $K_{\mathrm{p}}=7$ ) (Buonsanto, 1999; Desai \& Shah, 2018b). It has been observed from the $K_{\mathrm{p}}$ and $D_{\mathrm{st}}$ plot (refer Fig. 3) that intense geomagnetic storm $\left(D_{\mathrm{st}}-124, K_{\mathrm{p}}=8\right)$ was present on the day of 8 September 2017. It can be seen from the changes in the indices that the storm began on 7 September 2017 at approximately 22 UT (16.30 LT) and ended at 04 UT on 9 September 2017. In (Desai \& Shah, 2018b), a study was made to the various geomagnetic parameters and the impact of intense geomagnetic storm on the NavIC/IRNSS system with detailed. 


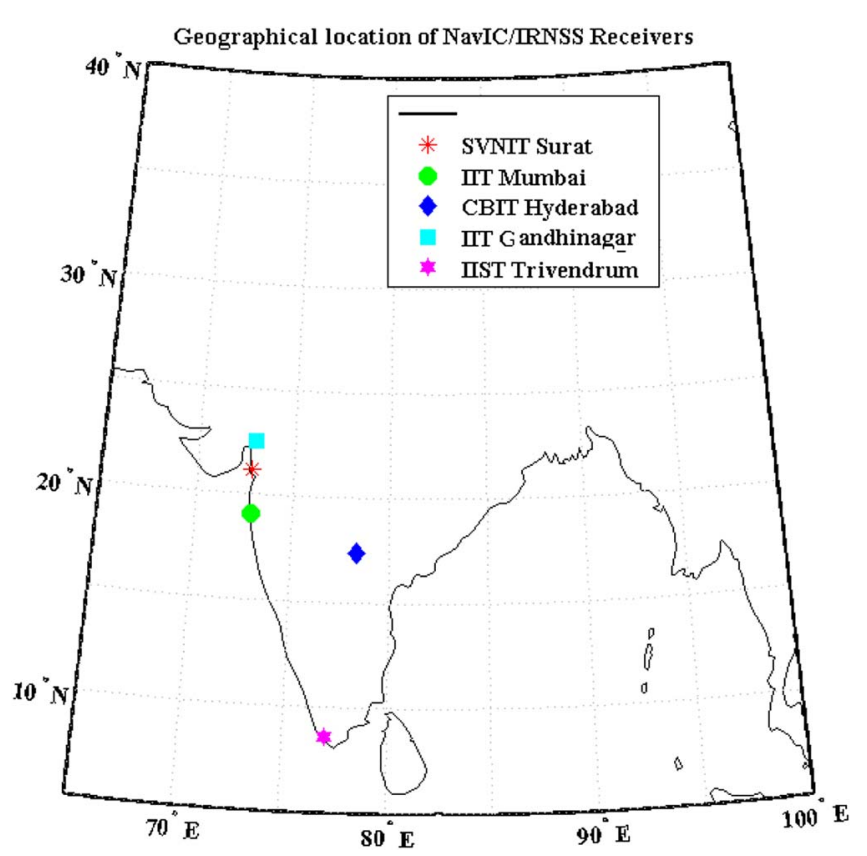

Fig. 4. Observational geographical locations of NavIC/IRNSS receivers.

The raw data from 3 September 2017 (Time of Week Count [TOWC] $=0$ ) to 9 September 2017 (TOWC $=648,000)$, of the dual-frequency NavIC/IRNSS receivers for the SVNIT Surat $\left(21.16^{\circ} \mathrm{N}, 72.78^{\circ} \mathrm{E}\right)$, IIT Bombay $\left(19.03^{\circ} \mathrm{N}, 72.91^{\circ} \mathrm{E}\right), \mathrm{CBIT}$ Hyderabad $\left(17.39^{\circ} \mathrm{N}, 78.31^{\circ} \mathrm{E}\right)$, IIT Gandhinagar $\left(22.52^{\circ} \mathrm{N}\right.$, $\left.72.92^{\circ} \mathrm{E}\right)$ and IIST Trivandrum $\left(8.62^{\circ} \mathrm{N}, 77.03^{\circ} \mathrm{E}\right)$ locations were collected. The geographical location of data collection NavIC/ IRNSS receiver stations are depicted in Figure 4. The IIST Trivandrum is located nearer to extended equatorial region, where another four stations located in EIA region.

The data for the Sardar Vallbhbhai National Institute of Technology (SVNIT), Surat (21.16 Lat, 72.78 Long) is acquired by the Accord NavIC/IRNSS + GPS receiver, which is located at the Communication Research Laboratory of Electronics Engineering Department. The NavIC/IRNSS satellites data for the location of IIT Bombay, CBIT Hyderabad, IIT Gandhinagar and IIST Trivandrum are provided by SAC, ISRO Ahmadabad. Initially, the distance between the NavIC/IRNSS satellites $(1 \mathrm{~A}-1 \mathrm{G})$ and the user receiver is calculated by extracting the time to travel information from the raw data. After calculating the ranges or distances for the single satellite, $\mathrm{L}_{5}$-band ionodelay is measured using the dual-frequency approach presented in equation (4). Here, the ionodelay for single frequency NavIC/IRNSS is computed using regional GIVE model (Desai $\&$ Shah, 2018a) and proposed local TSE model. Finally, the performance of single frequency models is validated by computing the residual error mentioned in equation (16).

\subsection{Case-2}

Here, TSE algorithm analyzes by considering two separate NavIC/IRNSS receivers. As shown in Figure 5, the reference station (marked with pink color) is selected as the TSE

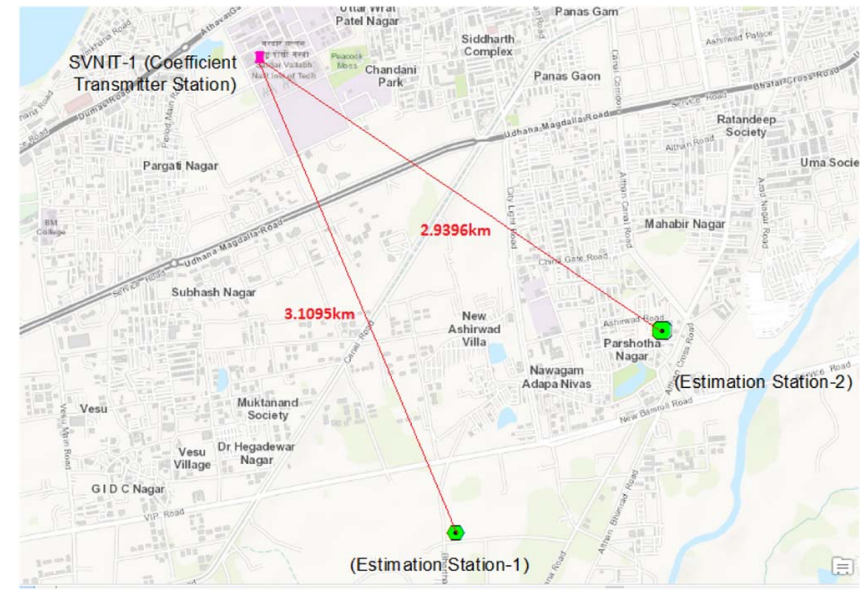

Fig. 5. Station physical location in ArcMap10.3.

coefficient generated station, which is located at the Communication Research Laboratory, Electronics Engineering Department, SVNIT, Surat (21.16392450 Lat., 72.78367420 Lon.) and the rover stations marked by green color are used for the analysis. The rover station use these TSE coefficients to estimate the total electron content and thereby the ionodelay. As depicts in Figure 5, the direct distance between SVNIT and Station 1 (21.139890 Lat, 72.794350 Lon) is approximately $3.1 \mathrm{~km}$ (ArcMap 10.3), and for station 2 (21.150223 Lat, 72.805590 Lon) is approximately $2.9 \mathrm{~km}$ (ArcMap 10.3). The quiet and disturbed days data are selected for the analysis, which is usually available for both receivers. In order to verify the single frequency global Klobuchar, regional GIVE and local TSE models performance is correlated with the reference dual frequency model in terms of ionodelay as well as positional accuracy (3DRMS, CEP and SEP). Result analysis has been carried out using the software tool MATLAB 2014 included in the next section.

\section{Simulation and results discussion}

This section validates the theoretical analysis of the models described in Section 2 and procedure explained in Section 3. The simulation tool MATLAB R.14 is used to estimate the ionodelay and $3 \mathrm{D}$ positional error for the NavIC/IRNSS $\mathrm{L}_{5}$-band.

\subsection{Results analysis for case-I}

The Figure 6 depicted the ionodelay comparison of six NavIC/IRNSS (1B-1G) satellites, measured by dual frequency approach at about five different geographical locations (Fig. 4) on the quiet day $\left(04 / 09 / 17, K_{\mathrm{P}}=3+, A_{\mathrm{P}}=17\right)$ (http://www. swpc.noaa.gov/phenomena/coronal-mass-ejections). It has been observed that for the all location the satellites $1 \mathrm{D}$ and $1 \mathrm{G}$ suffered more ionodelay compared to remaining NavIC/IRNSS satellites. The maximum ionodelay value of $1 \mathrm{G}$ NavIC/IRNSS satellite for the geographical location, IIST Trivendrum, CBIT Hyderabad, IIT Bombay, SVNIT Surat and IIT Gandhinagar are 16, 22, 27, 28 and $35 \mathrm{~m}$ respectively. Therefore it has been 


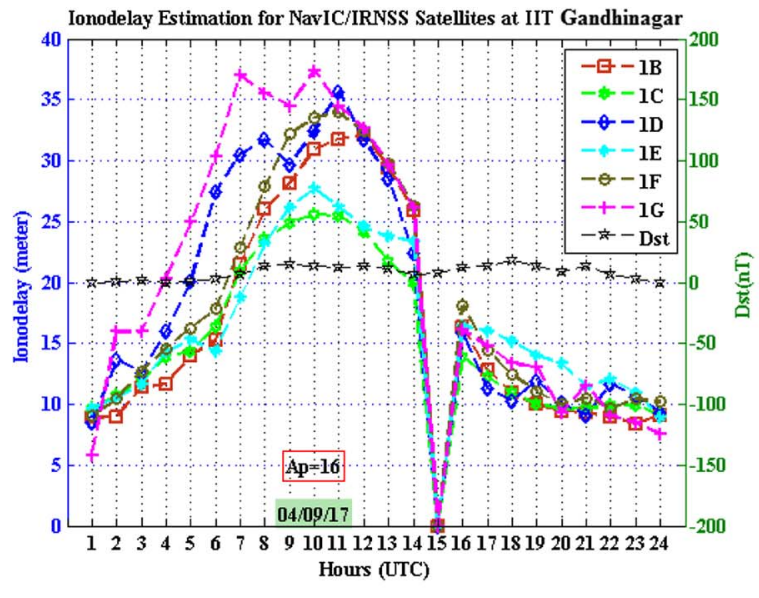

6(a)IIT Gandhinagar

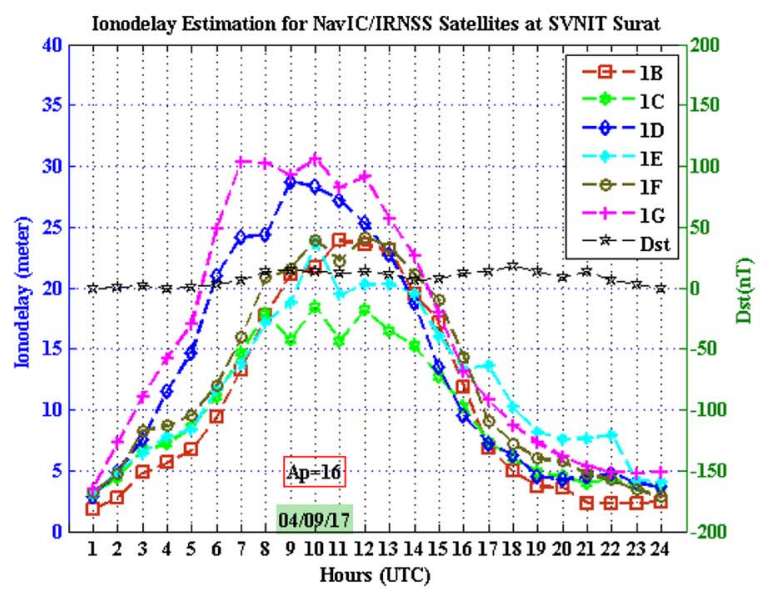

6(b)SVNIT Surat

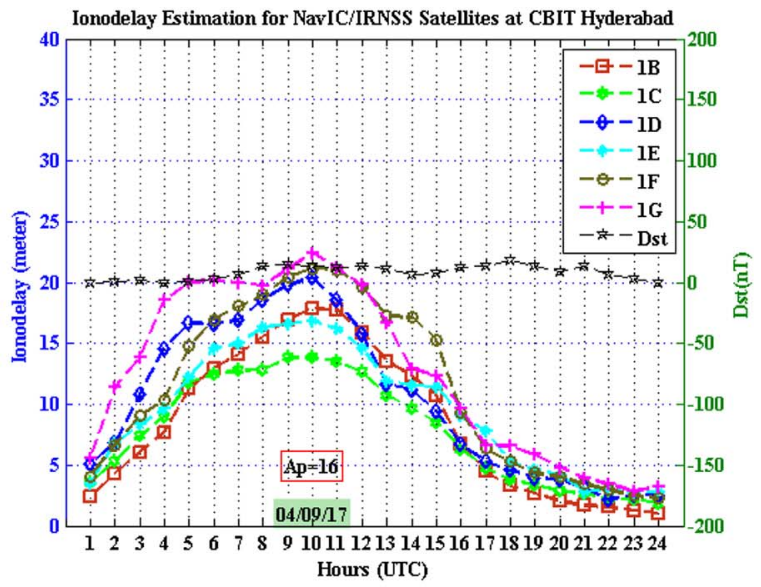

6(d)CBIT Hyderabad

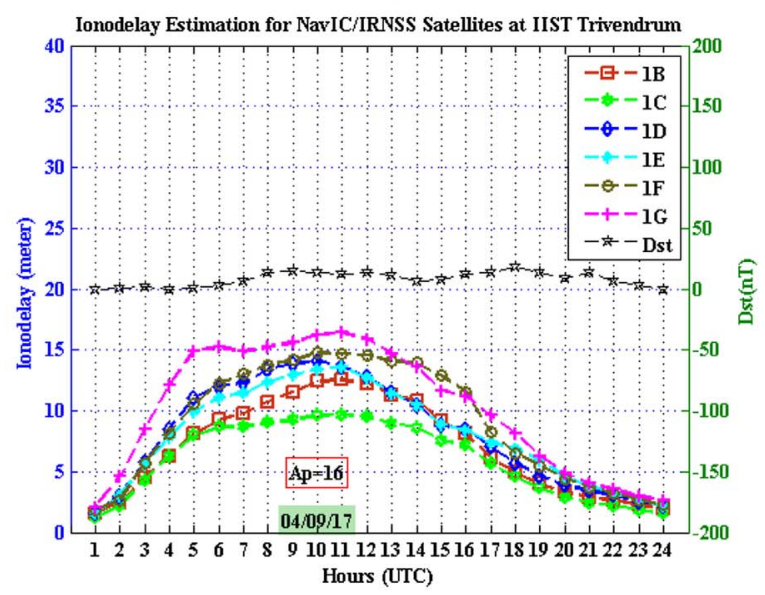

6(e)IIST Trivendrum

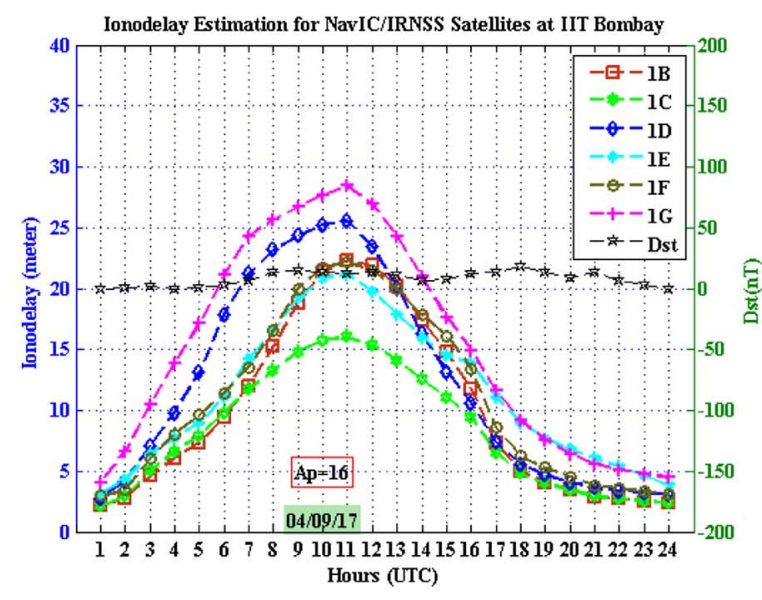

6(c)IIT Bombay

Fig. 6. Dual frequency ionodelay comparison of NavIC/IRNSS 1B-1G satellites for observed geographical location.

deduced that as the latitude is increased the ionodelay is also increased. For case-I, the local TSE algorithm is applies to all locations considering one week data from 3 to 9 September 2017.
The ionodelay performance comparison of the local TSE, dual-frequency and GIVE models of the NavIC/IRNSS six satellites at IIT Gandhinagar for the observation period (3-9 September 2017) is depicted in Figure 7. Here, the first 

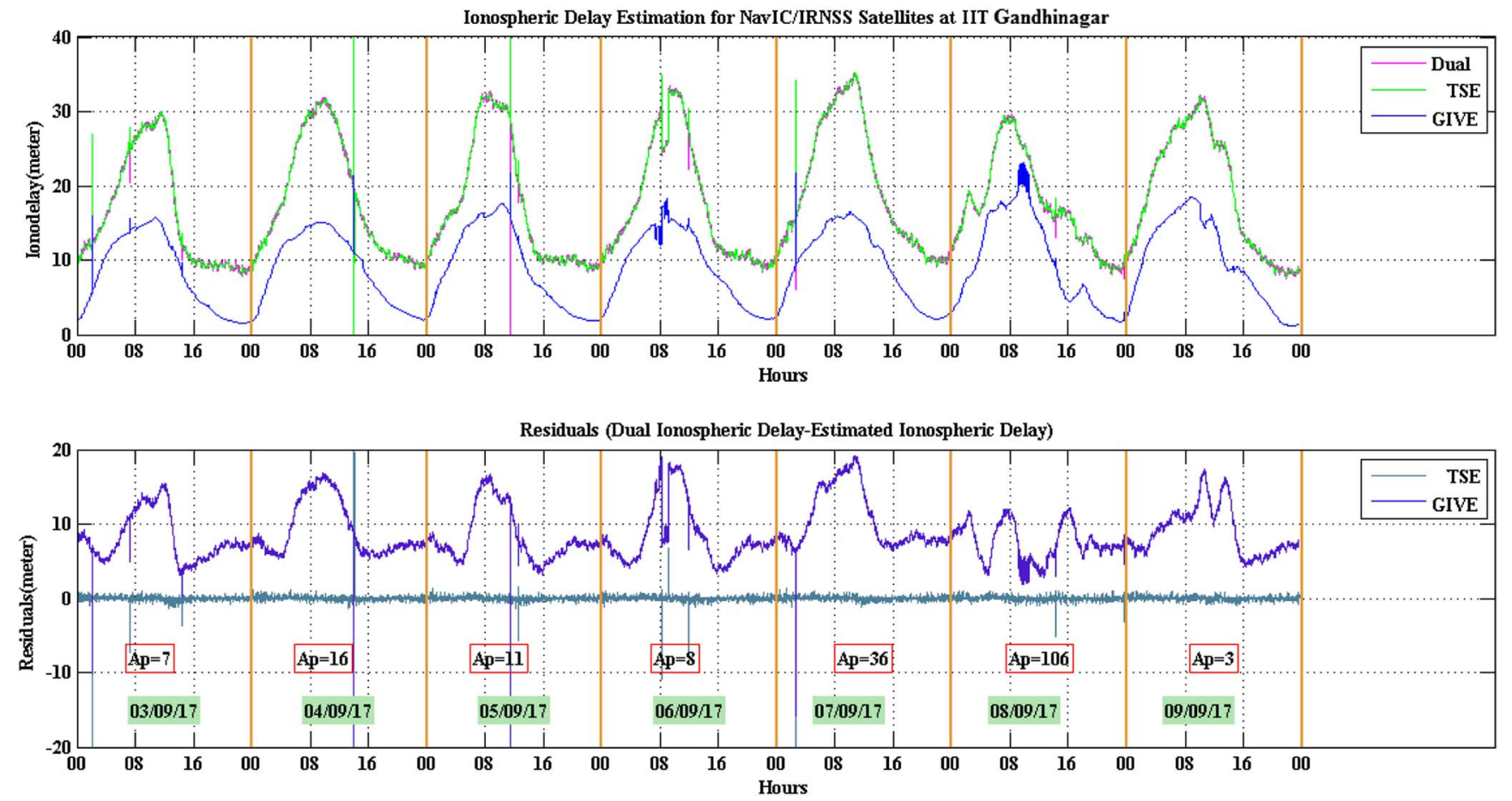

Fig. 7. The average ionodelay performance comparisons of TSE, dual frequency and GIVE model for NavIC/IRNSS satellites at the IIT Gandhinagar on the observation duration (3-9 September 2017).

ionodelay is computed for all the active NavIC/IRNSS satellites individually and then average ionodelay computed for the comparison. It has been observed that due to the existence of some multipath or noise, in IIT Gandhinagar (refer Fig. 7), the residual error is more for the regional GIVE model compared to the local TSE model. Hence, the performance of the GIVE model does not match with the reference dual frequency measurements but the local TSE model is following the same characteristics. For, IIT Gandhinagar, 8-10 m less ionodelay was measured on a geomagnetic storm day compared to another observation days.

As can be inferred from Figure 8, at SVNIT Surat the ionodelay is plotted for the duration of TOWC $=47,290$ (3 September 2017, UTC $=13: 08: 10$, LT $=18: 38: 10)$ to TOWC $=584,267(9$ September 2017, UTC $=18: 17: 47$, $\mathrm{LT}=23: 47: 47$ ), compared to other observation days, to the intense geomagnetic stormy day more deviation in ionodelay is observed and the performance of the local TSE is almost the same as that of the dual frequency. The GIVE model is estimated approximately 1-4 m less ionodelay compared to reference dual frequency measurement on the stormy. The residual error compares less for the local TSE model to regional GIVE model.

Similarly, the average ionodelay performance comparison of NavIC/IRNSS satellites for the geographical location IIT Bombay, CBIT Hyderabad and IIST Trivendrum are depicted in Figures 9-11 respectively. Figure 9 depicts the ionodelay comparison for the IIT Bombay for the duration of TOWC $=$ 0-560,229 (3 September 00 h UTC-9 September 11:36 h UTC). It has been deduced that at IIT Bombay, compared to other observation days, to the geomagnetic storm day approximately 1-3 m less ionodelay is estimated. Here also the performance of the local single frequency TSE is almost the same as that of the dual frequency model.

As can be seen from the Figure 10, at CBIT Hyderabad, compared to other observation days, to the geomagnetic stormy day measurement has a more delay of about $2 \mathrm{~m}$ and the performance of the TSE is almost the same as that of the dual frequency. The GIVE model has estimated approximately 1-4 m more ionodelay compared to reference dual frequency model in the UTC time of $8-12 \mathrm{~h}$. Similarly, it has been found that at the lower latitude IIT Trivendrum ionodelay estimated by the local TSE, GIVE and dual frequency model is nearly the same on all the quiet as well as disturbed days. However, the residual error shows that local TSE modem perform as nearly as dual frequency model compared to regional GIVE model.

The average ionodelay of five observational days (4-8 September 2017) estimated by Dual, GIVE and TSE models and their statistical performance compared in term of mean, median and standard deviation for five geographical locations are compared in Figure 12, which shows that local TSE model performance is same as dual frequency model. For IIT Gandhinagar, the highest mean, median and standard deviation and lowest for the low latitude IIST trivendrum station.

Therefore, for case-I, by observing the results of all observed geographic locations, it can be concluded that the single frequency local TSE model works nearly same as dual frequency model even in the presence of the intense geomagnetic storm. 

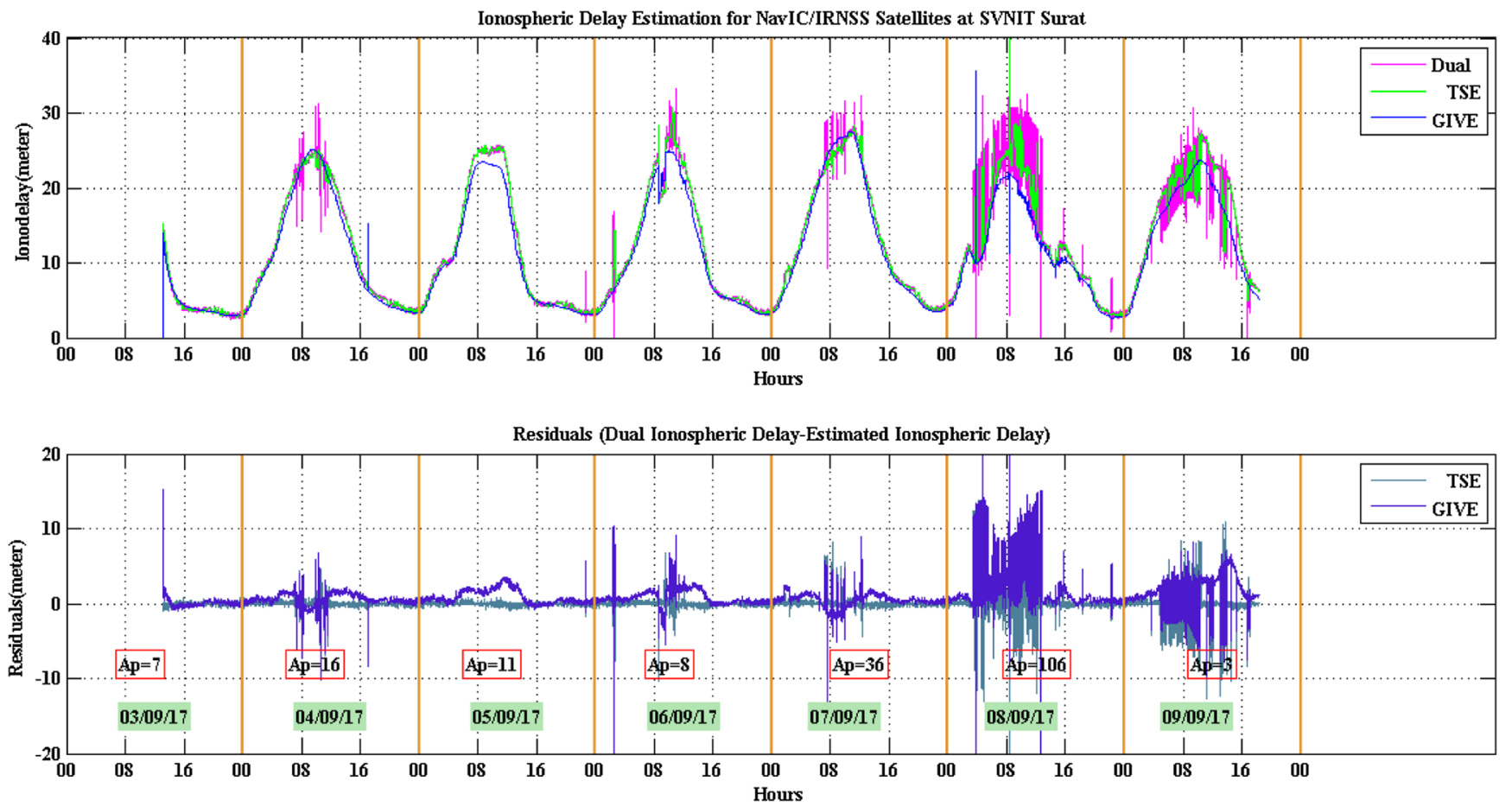

Fig. 8. The average ionodelay performance comparisons of TSE, dual frequency and GIVE model for NavIC/IRNSS satellites at the SVNIT Surat on the observation duration (3-9 September 2017).
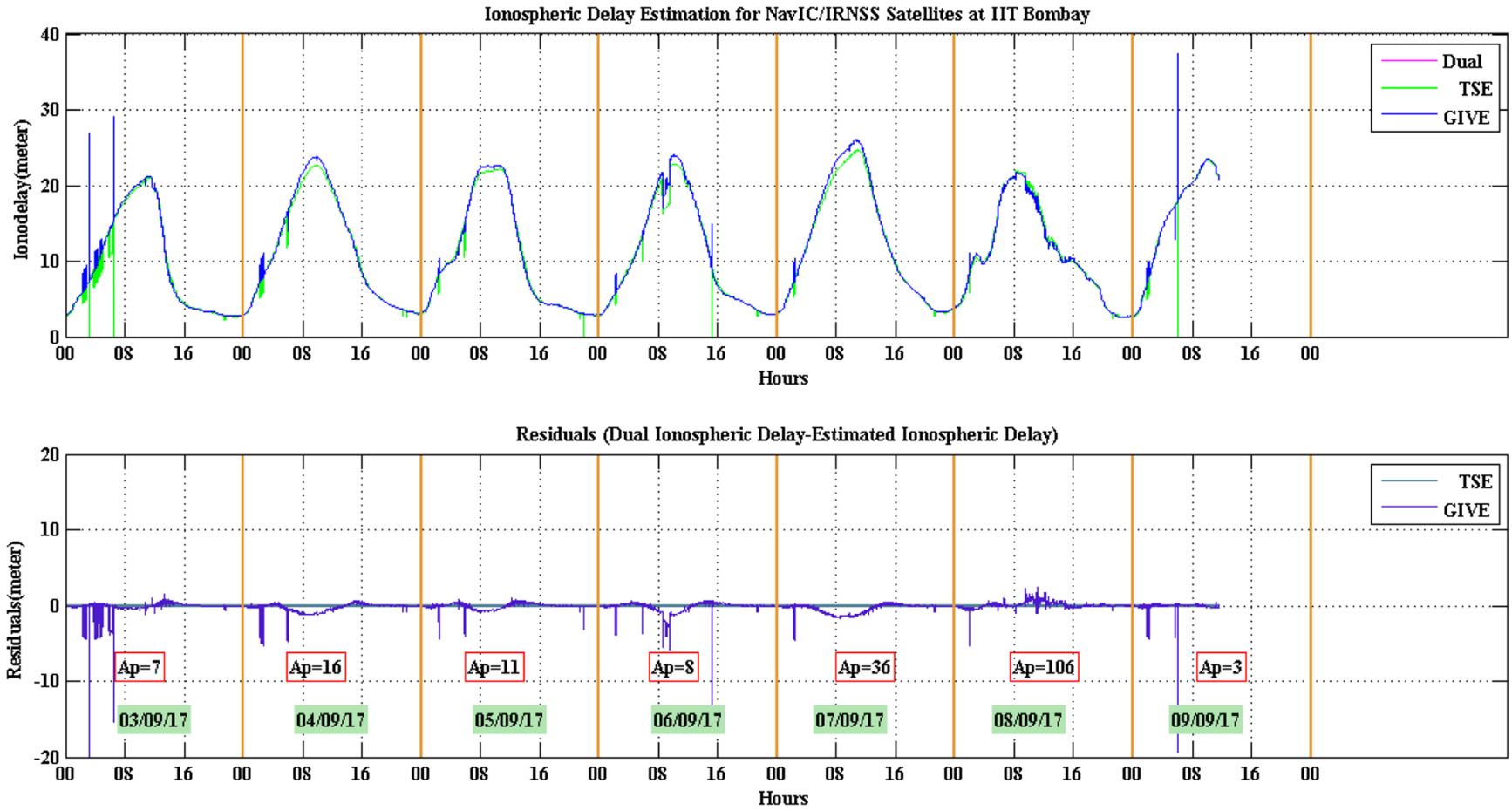

Fig. 9. The average ionodelay performance comparisons of TSE, dual frequency and GIVE model for NavIC/IRNSS satellites at the IIT Bombay on the observation duration (3-9 September 2017). 

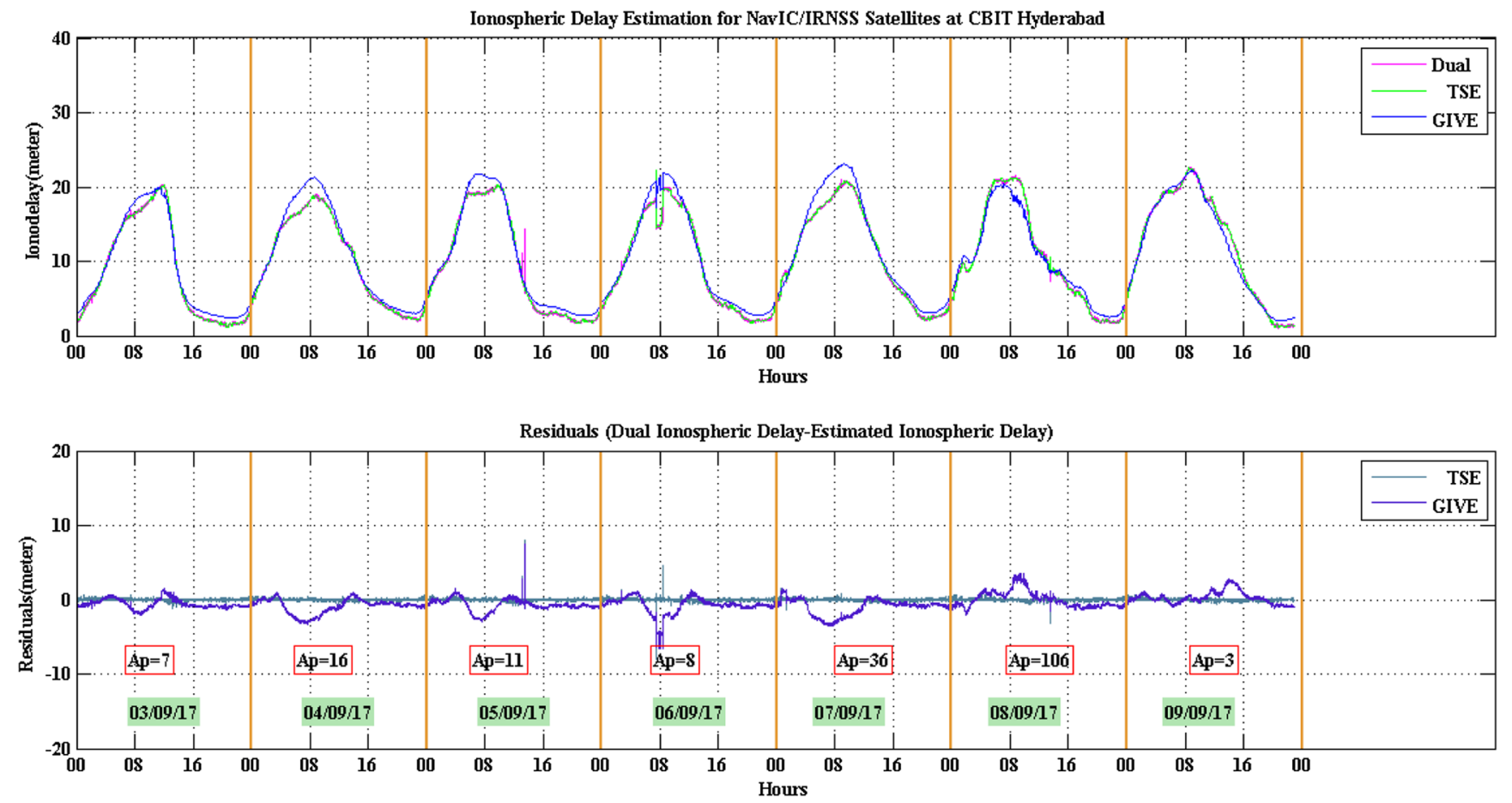

Fig. 10. The average ionodelay performance comparisons of TSE, dual frequency and GIVE model for NavIC/IRNSS satellites at the CBIT Hyderabad on the observation duration (3-9 September 2017).
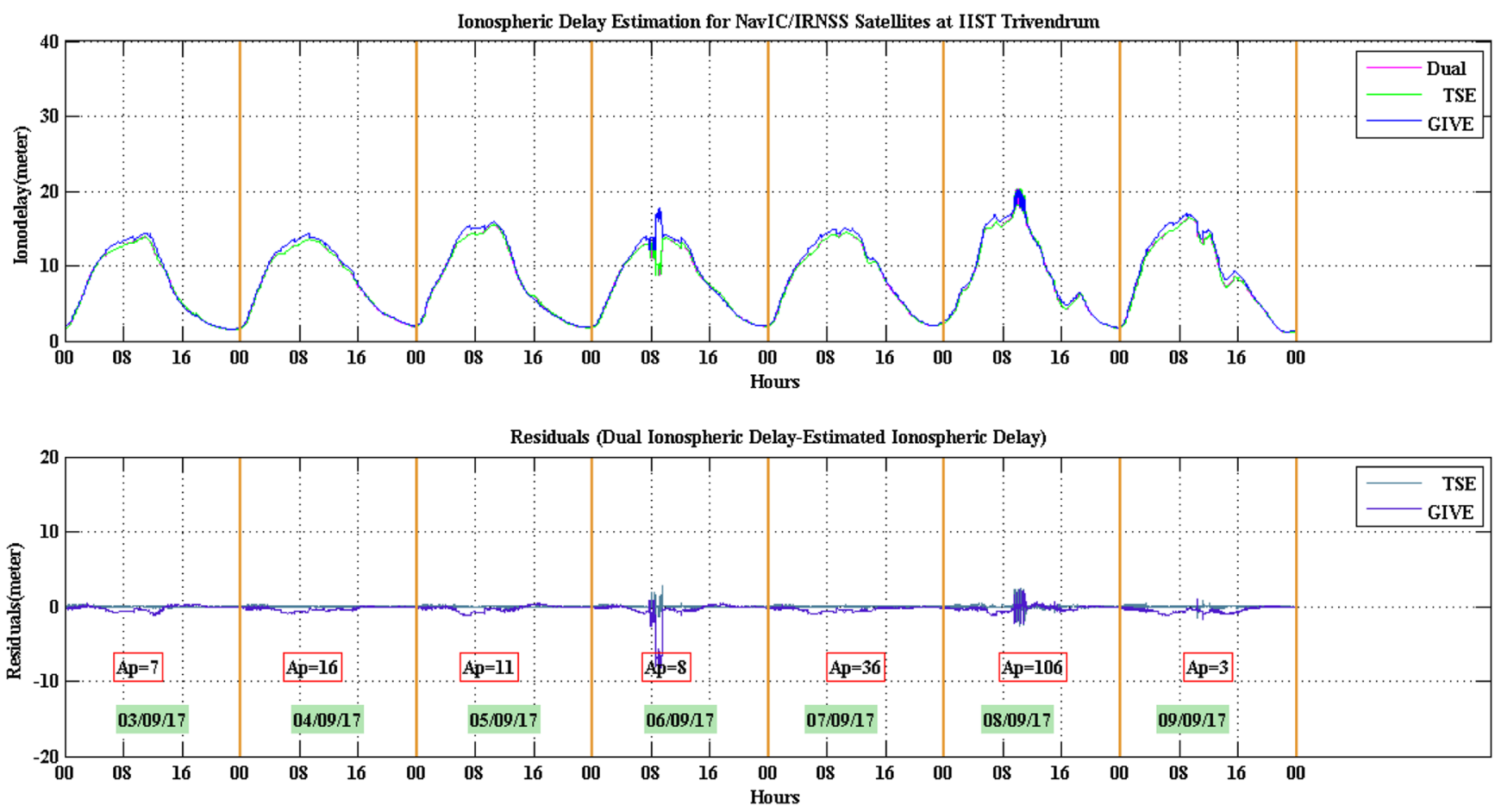

Fig. 11. The average ionodelay performance comparisons of TSE, dual frequency and GIVE model for NavIC/IRNSS satellites at the IIST Trivendrum on the observation duration (3-9 September 2017). 


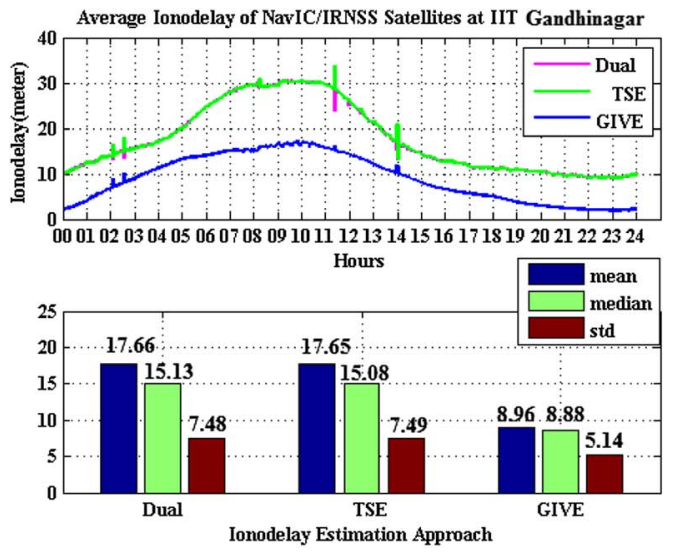

12(a)IIT Gandhinagar

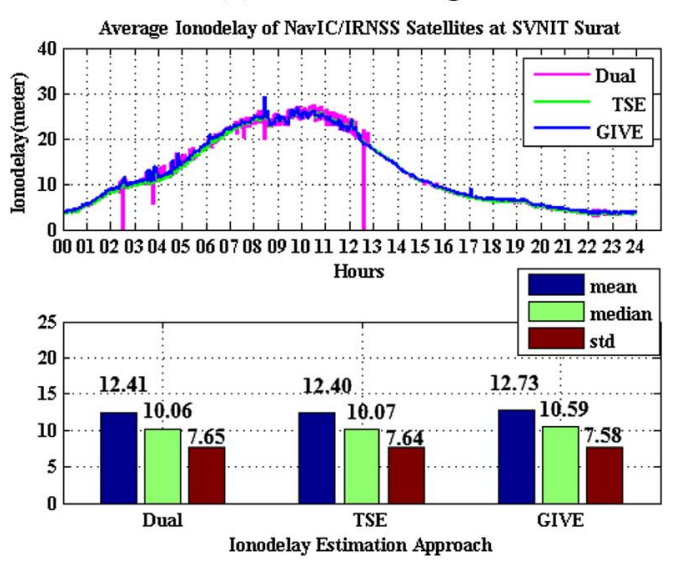

12(b)SVNIT Surat

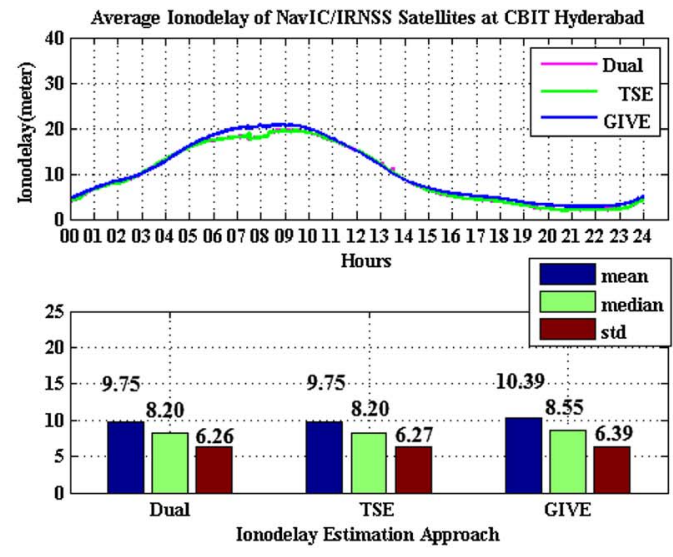

12(d)CBIT Hyderabad

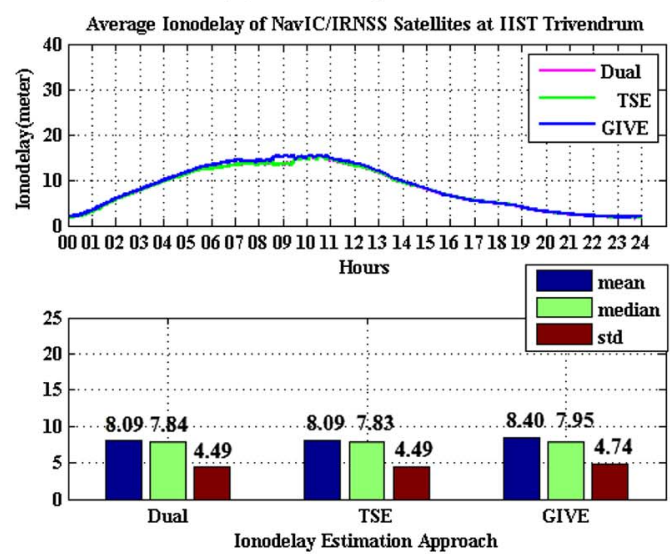

12(e)IIST Trivendrum

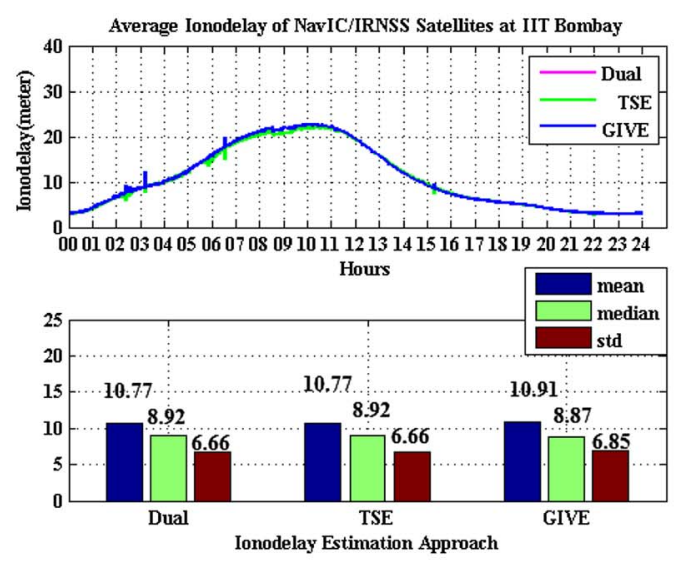

12(c)IIT Bombay

Fig. 12. The statistical performance comparison of various ionodelay models applied for NavIC/IRNSS satellites at observed geographical location.

\subsection{Results analysis for case-II}

For case-II, we choose two NavIC/IRNSS receivers located as shown in Figure 5 with the same specifications with their corrected biases like clock corrections and clock drift etc., available from SATB L5/S.csv and RNBB.csv files of dual frequency Accord NavIC/IRNSS receivers. Firstly, we verify the performance of the local TSE at both the rover stations and estimated the ionodelay as well as the position of the rovers to.

The ionodelay is calculated at rover station 1 , from the disturbed day $\left(04 / 09 / 16, K_{\mathrm{P}}=4+, A_{\mathrm{P}}=27\right.$ and $D_{\mathrm{st}}=$ below $-40 \mathrm{nT}$ ) by the local TSE model for six NavIC/IRNSS satellites 


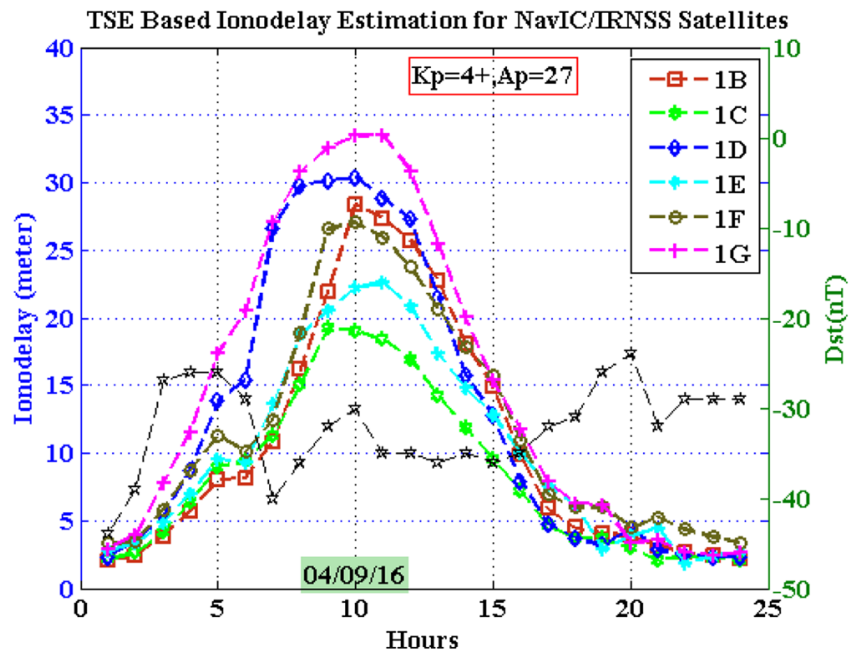

Fig. 13. TSE based ionodelay estimation for NavIC/IRNSS satellites.

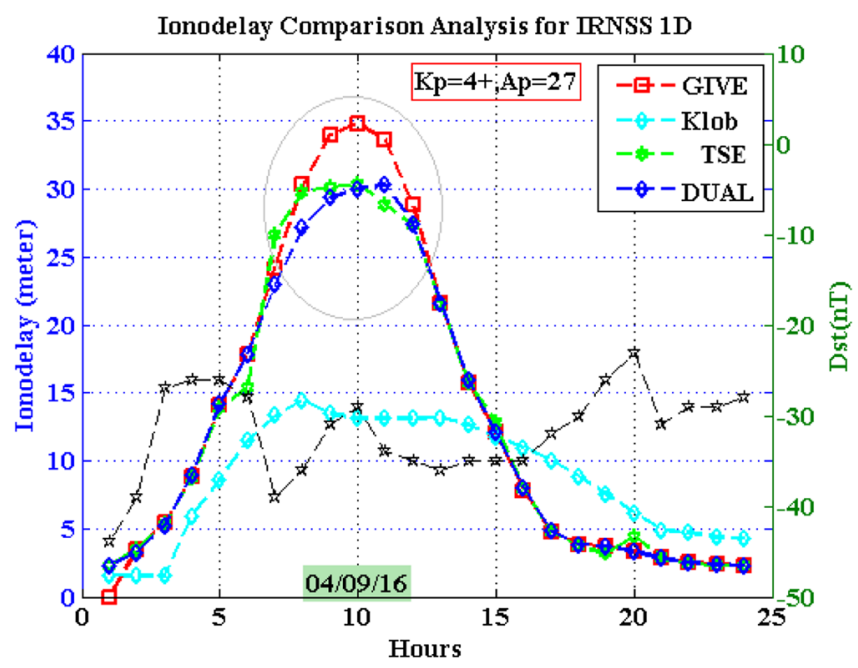

Fig. 14. Ionodelay estimation comparison for NavIC/IRNSS 1D satellites.

$(1 \mathrm{~B}-1 \mathrm{G})$ is depicted in Figure 13. Here, the day for the analysis is selected based on the data availability of two NavIC/IRNSS receivers within the $10 \mathrm{~km}$ region. Similar to case-I (refer Fig. 6), we observed that for the disturbed day the satellites $1 \mathrm{D}$ and $1 \mathrm{G}$ suffered more compared to other NavIC/IRNSS satellites. Therefore, for further performance comparison between single frequency models (global Klobuchar, region GIVE and local TSE) and the dual frequency model, the results analysis of NavIC/IRNSS 1D (Fig. 14) and 1G (Fig. 15) satellites are plotted as a reference.

It is observed that the local TSE and the dual frequency provide similar performance, where the former utilizes single frequency while the later utilizes two frequencies. In addition, the global eight-coefficient Klobuchar model effectively evaluated only $50 \%$ of ionodelay and the grid-based regional GIVE model performance is not matched with the reference dual frequency model (grey circles in Figs. 14 and 15 respectively).

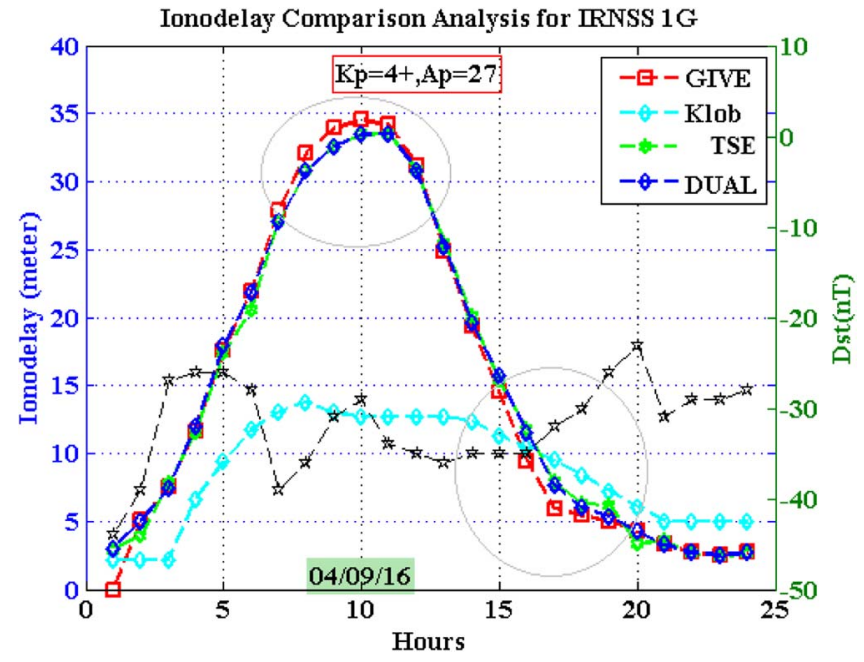

Fig. 15. Ionodelay estimation comparison for NavIC/IRNSS $1 \mathrm{G}$ satellites.

The same observation is made for the remaining NavIC/IRNSS satellites as well.

To analyze the effect of ionodelay on NavIC/IRNSS $\mathrm{L}_{5}$-band, the 3D position is calculated by the ILS estimation algorithm (He \& Bilgic, 2011). For optimization of position, the 3D positional error in terms of Earth Center Earth Fix (ECEF) coordinates is measured after correcting the ionospheric effect, tropospheric effect and the receiver all hardware and satellite clock biases. Here, the troposphere delay for the NavIC/IRNSS $\mathrm{L}_{5}$-band signal is estimated by the Hopfield model (Hopfield, 1971). The NavIC/IRNSS 3D positional error in term of the East, North and Up coordinate system are estimated for the combination with (I) the dual frequency correction, (II) the global Klobuchar correction, (III) the regional GIVE correction, (IV) with the local TSE correction, and $(\mathrm{V})$ the augmented NavIC/IRNSS with GPS correction, which is depicted in the Figure 16a-e respectively.

The performance analysis is repeated for rover station 1 on $07 / 09 / 16\left(K_{P}=3+, A_{P}=12\right)$ for TOWC $1-55,000 \mathrm{~s}$. The position accuracy is evaluated in term of 3DRMS, CEP and SEP, which is encapsulated in Table 1. It has been observed that NavIC/IRNSS positional accuracy is better after applying ionospheric correction using single frequency local TSE model compared to global Klobuchar and regional GIVE model. Also, the performance of NavIC/IRNSS with TSE correction is nearly thesame as the dual correction with a difference in performance of approximately $0.2-0.8 \mathrm{~m}$ for 3DRMS, $0.1-0.3 \mathrm{~m}$ for CEP and $0.4-0.7 \mathrm{~m}$ for SEP.

The test is repeated at another rover station (Station 2 refer Fig. 5). The analysis is carried out for the quiet days, 13/08/17 $\left(K_{\mathrm{P}}=2+, A_{\mathrm{P}}=6, \mathrm{TOWC}=1-40,000 \mathrm{~s}\right)$ and $01 / 08 / 18\left(K_{\mathrm{P}}=1+\right.$, $\left.A_{\mathrm{P}}=3, \mathrm{TOWC}=1-71,327 \mathrm{~s}\right)$. The estimated 3D position statistical performance is shown in Table 2. Here, also it is noticed that local TSE model performance is nearly the same as dual frequency model in the local region. Therefore, in case-II of the results analysis of two rover stations, it has been inferred that the local TSE model worked efficiently same as dual frequency model by reducing the hardware cost of extra frequency. 


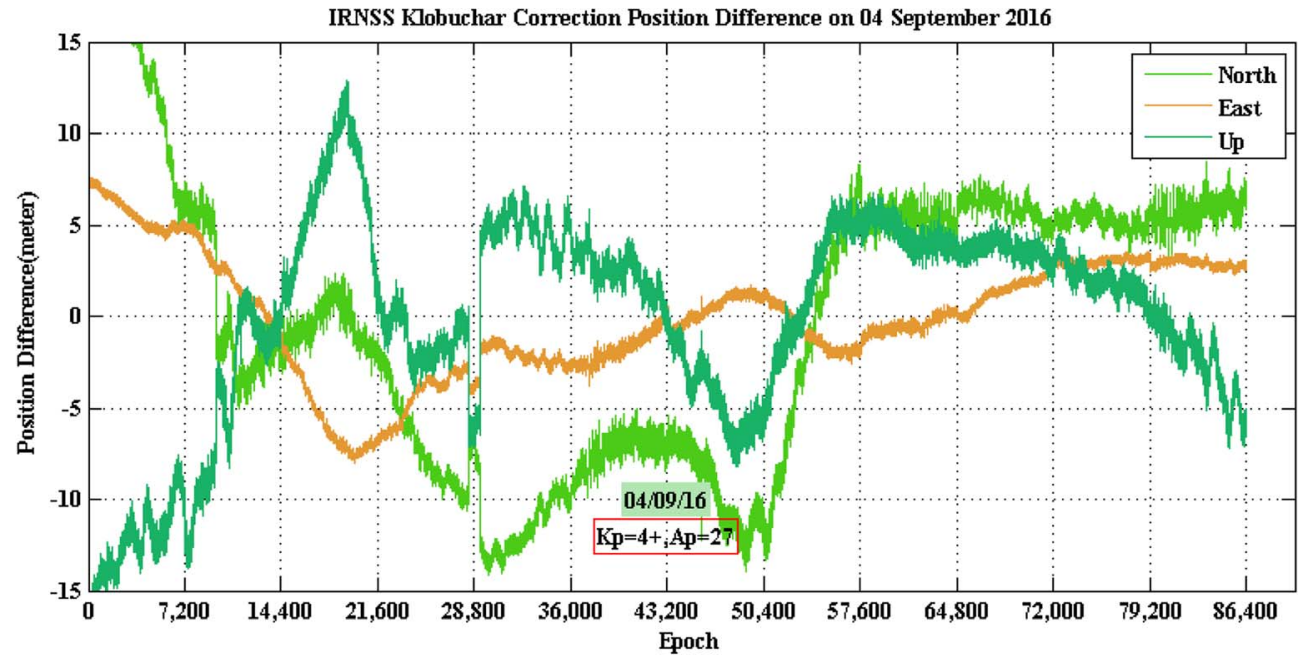

(a)

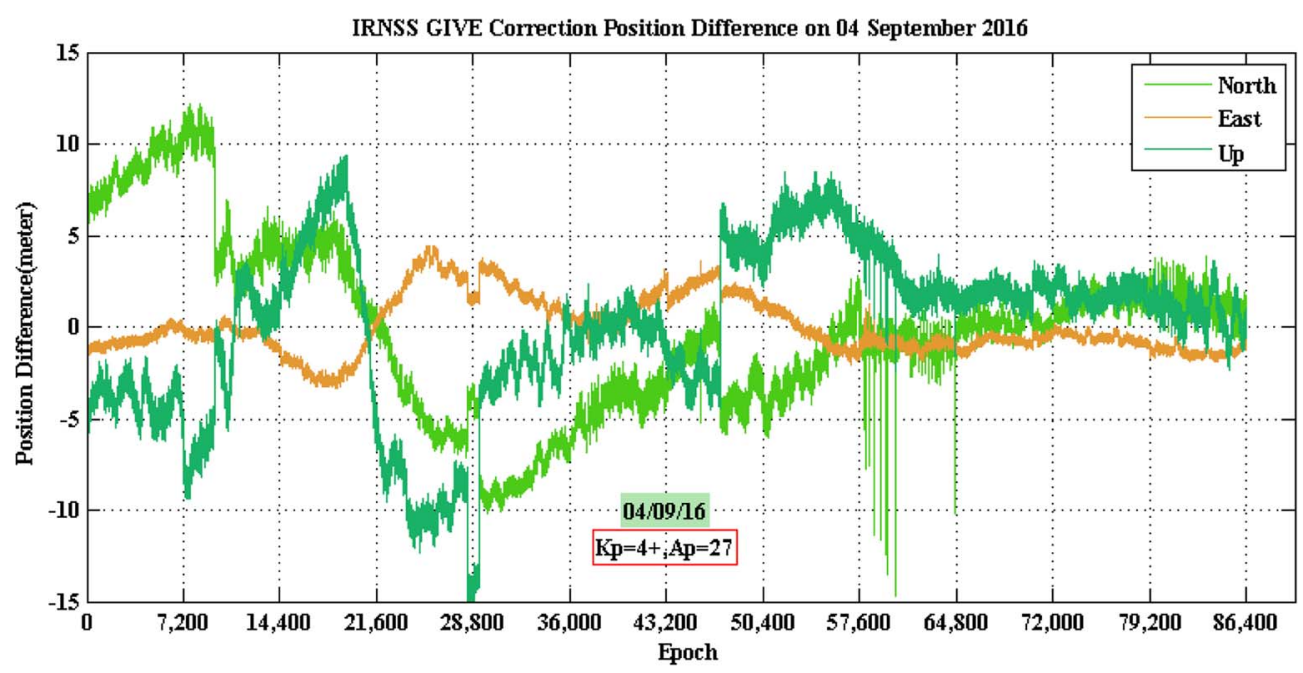

(b)

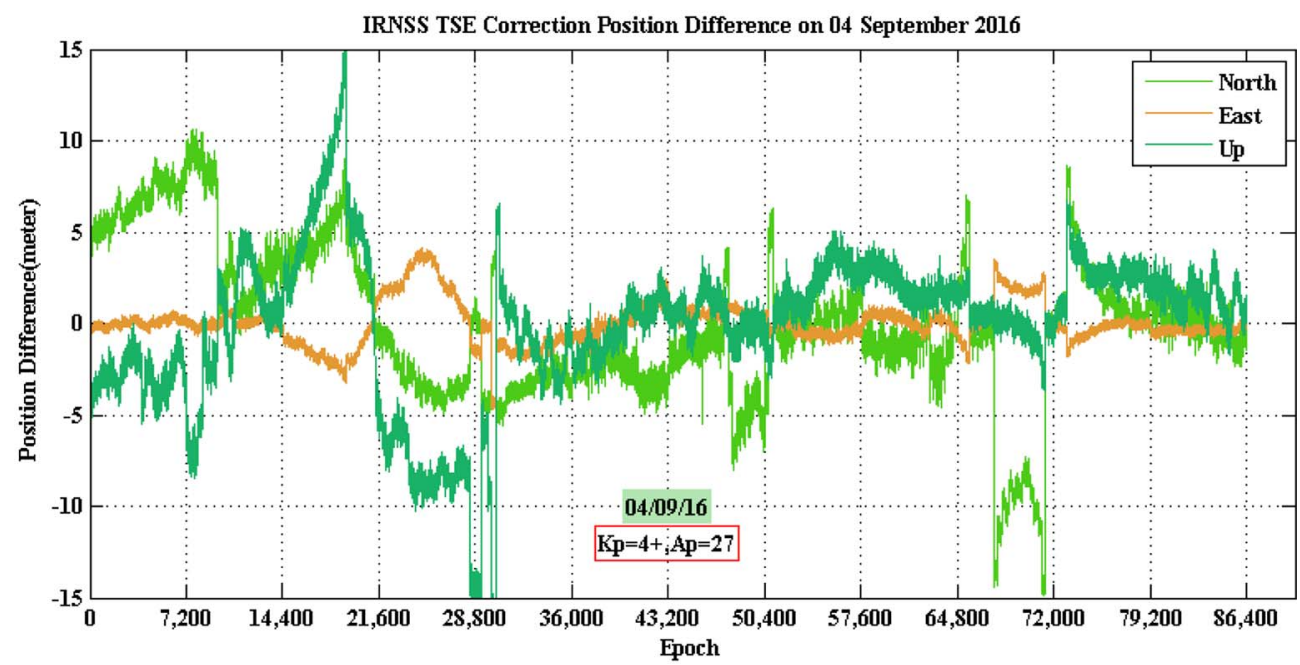

(c)

Fig. 16. Position Error (a) IRNSS with Dual frequency Correction, (b) IRNSS with Klobuchar Correction, (c) IRNSS with TSE Correction, (d) IRNSS with GIVE Correction, and (e) IRNSS + GPS Correction on 04/09/17. 


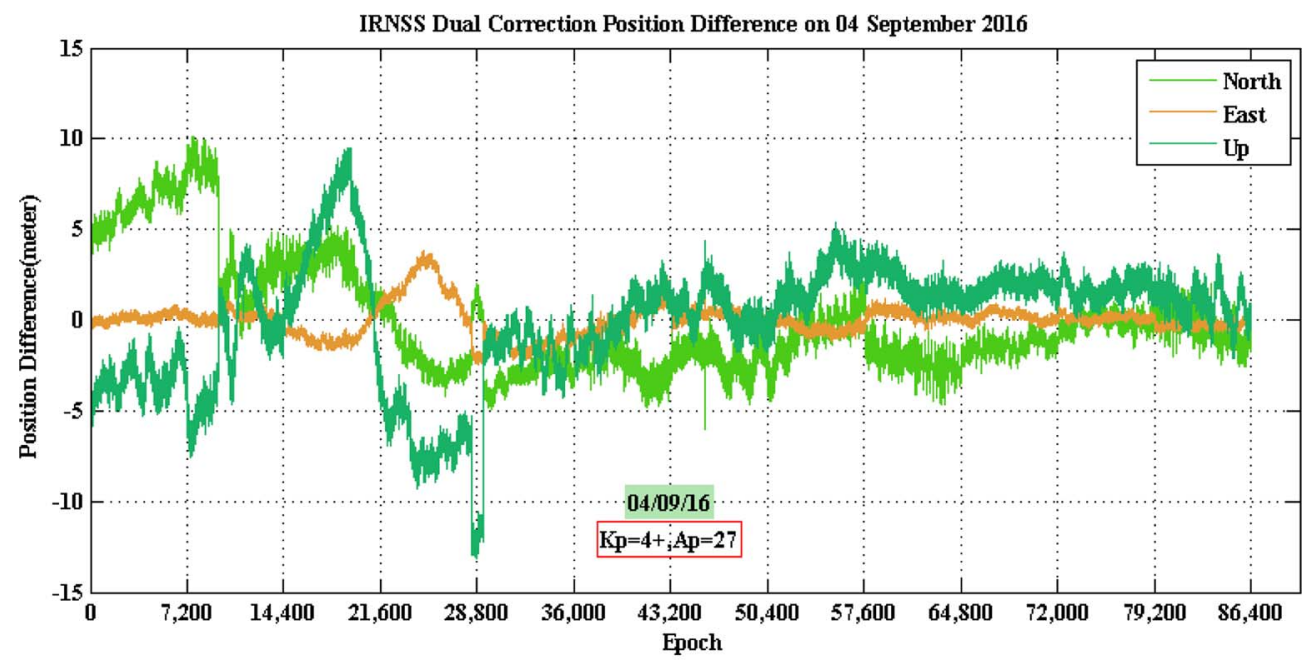

(d)

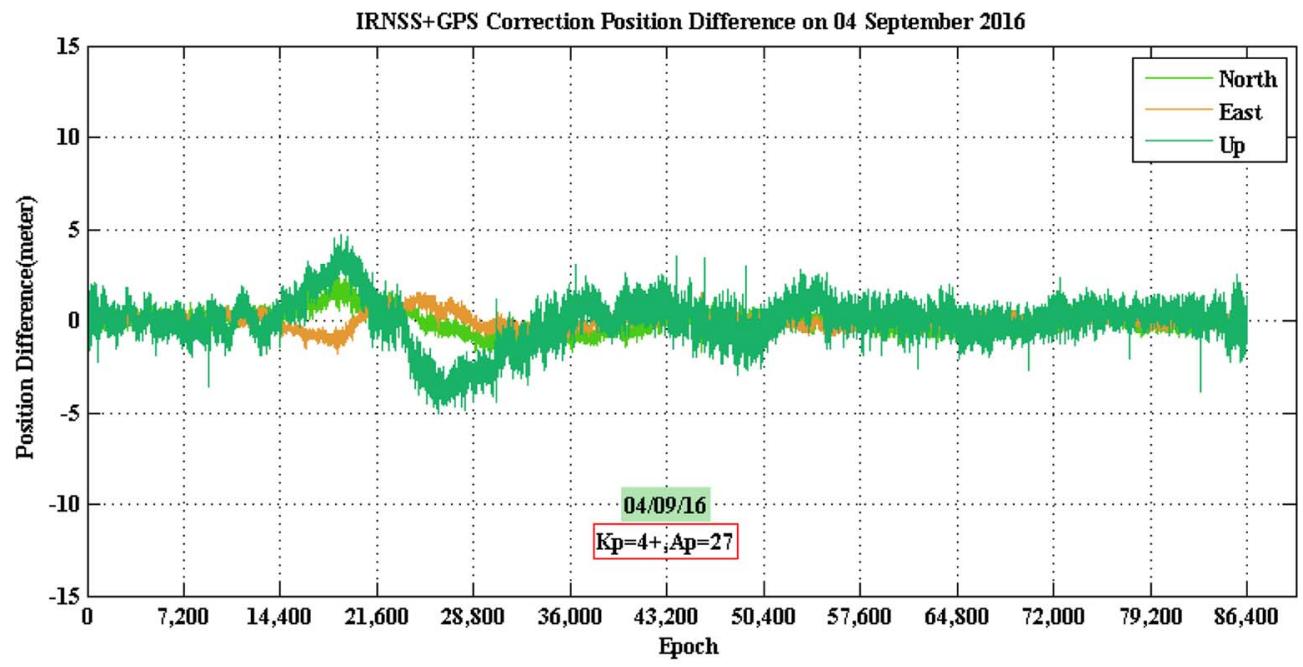

(e)

Fig. 16. Continued.

Table 1. The statistical positional error analysis at rover station 1 .

\begin{tabular}{|c|c|c|c|c|}
\hline Date $(\mathrm{dd} / \mathrm{mm} / \mathrm{yy})$ & Methods/Algorithm & 3DRMS (m) & $\mathrm{CEP}(\mathrm{m})$ & $\mathrm{SEP}(\mathrm{m})$ \\
\hline 04/09/16 & IRNSS Klobuchar & 22.9805 & 5.9001 & 6.7118 \\
\hline \multirow[t]{3}{*}{ (TOWC) $1: 86,400$ ) } & IRNSS-TSE with 5 min coefficients & 9.3718 & 2.4091 & 3.7223 \\
\hline & IRNSS Dual & 8.5396 & 2.1705 & 3.0530 \\
\hline & IRNSS Dual + GPS & 3.8683 & 1.0121 & 1.2468 \\
\hline 07/09/16 & IRNSS Klobuchar & 18.5805 & 4.5001 & 5.1728 \\
\hline \multirow{2}{*}{ (TOWC) $1: 55,000$} & IRNSS Dual & 6.7468 & 1.7676 & 2.8412 \\
\hline & IRNSS Dual + GPS & 2.6015 & 0.6665 & 0.8477 \\
\hline
\end{tabular}

Notes: 3DRMS, three dimensional distance root mean square; CEP, circular error probability; GIVE, grid ionospheric vertical error; GPS, global positioning system; IRNSS, Indian Regional Navigation Satellite System; SEP, spherical error probability; TOWC, time of week count; TSE, Taylor Series Expansion. 
Table 2. The statistical positional error analysis at rover station 2.

\begin{tabular}{|c|c|c|c|c|}
\hline Date(dd/mm/yy) & Methods/Algorithm & 3DRMS (m) & $\mathrm{CEP}(\mathrm{m})$ & SEP $(m)$ \\
\hline \multicolumn{5}{|c|}{ Station-2 (latitude of 21.150223 and longitude of 72.805590 ) } \\
\hline $13 / 08 / 17$ & IRNSS Klobuchar & 16.2615 & 2.4301 & 2.1728 \\
\hline \multirow[t]{3}{*}{ (TOWC) $1: 40,000$} & IRNSS TSE 5 min coefficients & 3.5583 & 0.8485 & 1.3423 \\
\hline & IRNSS Dual & 2.7021 & 0.7508 & 0.8396 \\
\hline & IRNSS Dual + GPS & 1.5215 & 0.4625 & 0.6172 \\
\hline 08/01/18 & IRNSS Klobuchar & 22.5805 & 4.5001 & 5.1728 \\
\hline \multirow{2}{*}{ (TOWC) $1: 71,327$} & IRNSS Dual & 6.6045 & 1.5714 & 1.9519 \\
\hline & IRNSS Dual + GPS & 3.2675 & 0.7665 & 0.8273 \\
\hline
\end{tabular}

Notes: 3DRMS, three dimensional distance root mean square; CEP, circular error probability; GIVE, grid ionospheric vertical error; GPS, global positioning system; IRNSS, Indian Regional Navigation Satellite System; SEP, spherical error probability; TOWC, time of week count; TSE, Taylor Series Expansion.
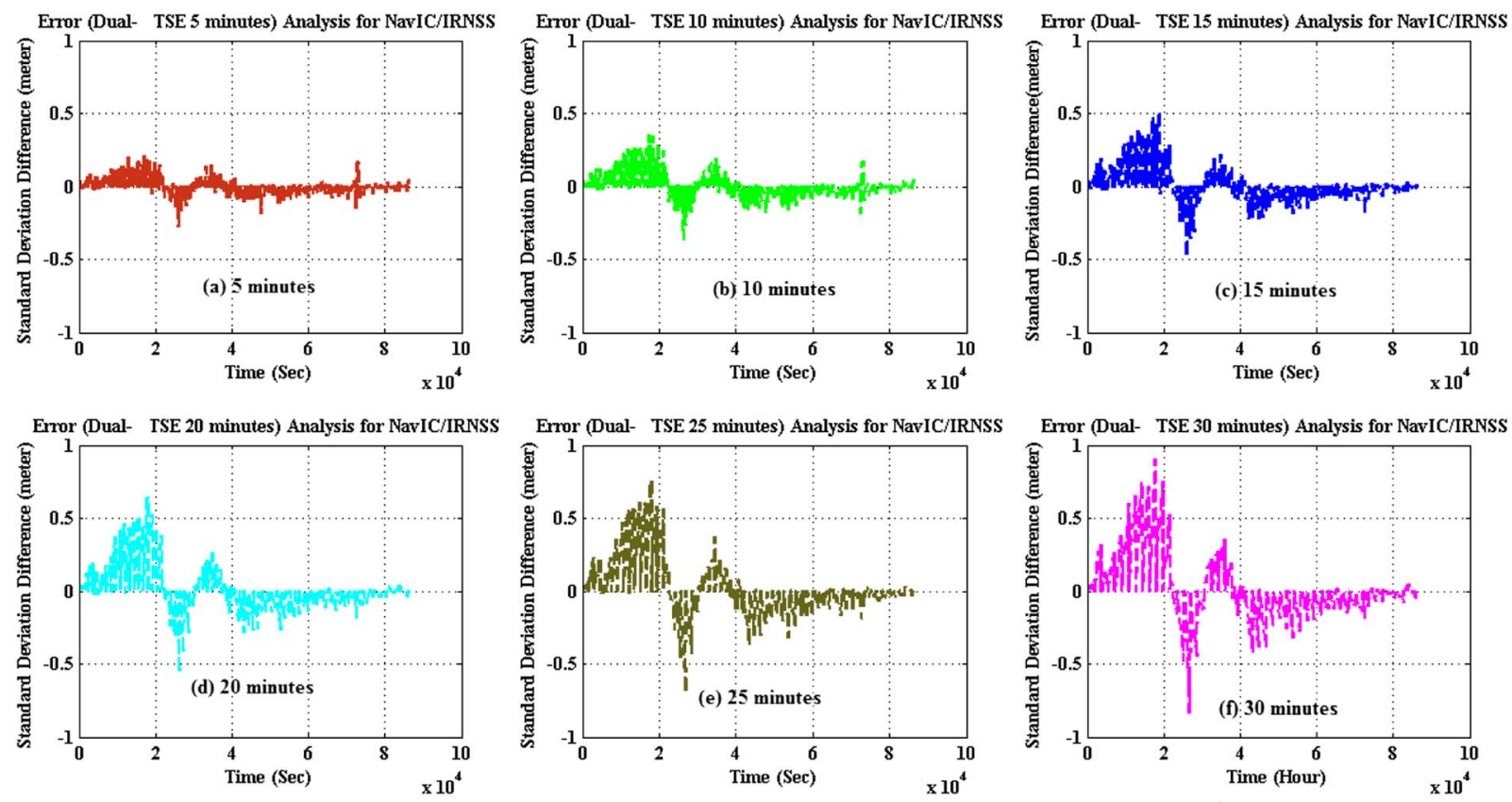

Fig. 17. TSE standard deviation error analysis for Broadcasting Interval (a) $5 \mathrm{~min}$, (b) $10 \mathrm{~min}$, (c) $15 \mathrm{~min}$, (d) $20 \mathrm{~min}$, (e) $25 \mathrm{~min}$, and (f) $30 \mathrm{~min}$.

\subsection{Computational complexity}

Here, the cost of computational complexity is further reduced by increasing the TSE coefficient transmission duration from 5 to $30 \mathrm{~min}$. Figure 17 depicts the standard deviation error between the dual frequency measurements of the ionodelay and the estimated using TSE coefficients, which is broadcast at intervals of $5,10,15,20,25$ and $30 \mathrm{~min}$. It is noticed that the local TSE model can work effectively for up to $15 \mathrm{~min}$ of the broadcast interval if $0.5 \mathrm{~m}$ of deviation in the performance is accepted.

\section{Conclusions}

This paper provides a comparative analysis between single frequency local TSE and different ionospheric models to improve the positional accuracy of future NavIC/IRNSS systems. The coefficient generation and ionodelay estimation process are applied based on local TSE model for two cases considering, (I) a static receiver, and (II) two rover stations of the same kind placed a few kilometers apart. For case-I, the performance of TSE model is verified under the effect of intense geomagnetic storm (8 September 2017) at five geographical 
locations (IIST Trivendrum, CBIT Hyderabad, IIT Bombay, SVNIT Surat and IIT Gandhinagar). It has been observed from the residual errors the comparison that even in the presence of intense geomagnetic storm, the single frequency local TSE worked efficiently the same as the dual frequency model.

In case II, based on the broadcast TSE coefficients generated by the reference dual-frequency NavIC/IRNSS receiver of the SVNIT Surat station, the nearby (in the local $<10 \mathrm{~km}$ area) rover single frequency NavIC/IRNSS receivers (two locations) attempts to accurately estimate the ionodelay. The performance analysis of the single frequency, eight coefficient global Klobuchar model, the regional grid based GIVE model and local TSE models are compared with the reference dual frequency model. It has been observed from the comparative analysis of ionodelay, and $\mathrm{L}_{5}$-band positional accuracy (3DRMS, CEP and SEP) that compared to the global Klobuchar as well as the regional GIVE model the local TSE model performed best.

It is observed that the ionospheric correction applied by single frequency local TSE model has a $0.8 \mathrm{~m}$ of errors compared to the reference dual frequency model. Moreover, the local TSE that generated that coefficients every 5 and $10 \mathrm{~min}$ will nearly perform the same. Therefore, ionospheric correction applied by the local TSE model cannot only improves the performance of the rover NavIC/IRNSS receivers in the local region but will also reduce the computational cost and additional frequency if errors of up to $0.8 \mathrm{~m}$ are tolerated. This verification can be further extended at various geographical locations for the different atmospheric condition considered as a future research work.

Acknowledgements. This work is related to the research project "Study, Design and Simulation of Ionodelay effect based on Ionospheric Models and their correction techniques using Software Defined Radio" sponsor under the research program "Announcement of Opportunity for IRNSS/GAGAN Navigation Data Utilization Program" of Space Application Center, ISRO, Ahmadabad. The editor thanks Ernest Macalalad and an anonymous referee for their assistance in evaluating this paper.

\section{References}

Bhushana Rao GS. 2008. Ionospheric delay estimation for improving the global positioning system position accuracy. IETE J Res 54(1): 23-29. DOI: 10.1080/03772063.2008.10876178.

Buonsanto MJ. 1999. Ionospheric storms - A review. Space Sci Rev 88(8): 563-601. DOI: 10.1023/A:1005107532631.

Desai MV, Jagiwala D, Shah SN. 2016. Impact of dilution of precision for position computation in Indian regional navigation satellite system. In: IEEE Conference on Advances in Computing, Communications and Informatics (ICACCI), Jaipur, India, 21-24 September, pp. 980-986. DOI: 10.1109/ICACCI.2016.7732172.

Desai MV, Shah SN. 2015. Ionodelay models for satellite based navigation system. Afr J Comput ICT 8(2), 25-32.

Desai MV, Shah SN. 2017. Analysis of ionospheric correction approach for IRNSS/NavIC system based on IoT platform. In: International Conference on Future Internet Technologies and Trends, Springer Cham, Surat, India, 31st August-2nd September, pp. 174-183. DOI: 10.1007/978-3-319-73712-6_18.
Desai MV, Shah SN. 2018a. The GIVE ionospheric delay correction approach to improve positional accuracy of NavIC/IRNSS singlefrequency receiver. Current Science (00113891) 114(8): 1665 1676. DOI: $10.18520 / \mathrm{cs} / \mathrm{v} 114 / 108 / 1665-1676$.

Desai MV, Shah SN. 2018b. Impacts of intense geomagnetic storms on NavIC/IRNSS system. Ann Geophys 61(5): 557. DOI: 10.4401/ ag-7856.

Desai MV, Shah SN. 2019. An observational review on influence of intense geomagnetic storm on positional accuracy of NavIC/ IRNSS system. IETE Technical Review Paper, pp. 1-15. DOI: 10.1080/02564602.2019.1599739.

Fujita S, Kubo Y, Sugimoto S. 2009. Ionosphere total electron content estimation based on GNSS regression models at known positions. Int J Innov Comput Inform Contr 5(1): 139-152.

Fujita S, Yamamoto H, Iura T, Kubo Y, Sugimoto S. 2010. Modeling ionosphere VTEC over Japan based on GNSS regression models and GEONET. Int J Innov Comput Inform Contr 6(1): 155-169.

He Y, Bilgic A. 2011. Iterative least squares method for global positioning system. Adv Radio Sci: ARS 9: 203. DOI: 10.5194/ars9-203-2011.

Hopfield HS. 1971. Tropospheric effect on electromagnetically measured range: Prediction from surface weather data. Radio Sci 6(3): 357-367. DOI: 10.1029/RS006i003p00357.

Jagiwala DD, Shah SN. 2018. Impact of Wi-Fi interference on NavIC signal. Curr Sci 114(11): 2273-2280. DOI: 10.18520/cs/v114/i11/ 2273-2280.

Klobuchar JA. 1987. Ionospheric time-delay algorithm for singlefrequency GPS users. IEEE Trans Aerosp Electron Syst 3: 325-331. DOI: 10.1109/TAES.1987.310829.

Kumar PN, Sarma AD, Reddy AS. 2014. Modelling of ionospheric time delay of Global Positioning System (GPS) signals using Taylor Series Expansion for GPS Aided Geo Augmented Navigation applications. IET Radar Sonar Navig 8(9): 1081-1090. DOI: 10.1049/iet-rsn.2013.0351.

Macalalad EP, Tsai L-C, Wu J, Liu C-H. 2013. Application of the TaiWan Ionospheric Model to single-frequency ionospheric delay corrections for GPS positioning. GPS Solut Springer 17(3): 337-346. DOI: 10.1007/s10291-012-0282-8.

Misra P, Enge P. 2006. Global positioning system: Signals, measurements and performance, 2nd edn. Ganga-Jamuna Press, Lincoln, MA.

Ratnam DV, Sarma A. 2006. Modeling of Indian ionosphere using MMSE estimator for GAGAN applications. J Ind Geophys Uni $\mathbf{1 0}$ (4): 303-312.

Ratnam DV, Sarma A. 2012. Modeling of low-latitude ionosphere using GPS data with SHF model. IEEE Trans Geosci Remote Sens 50(3): 972-980. DOI: 10.1109/TGRS.2011.2163639.

Raveendran S, Desai MV, Shah SN. 2017. FFT averaging ratio algorithm for IRNSS. In: IEEE Conference on Advances in Computing, Communications and Informatics (ICACCI), Jaipur, India, 21-24 September, pp. 184-191. DOI: 10.1007/978-3-31973712-6_19.

Sarma A, Prasad N, Madhu T. 2006. Investigation of suitability of grid-based ionospheric models for GAGAN. Electron Lett IET 42 (8): 478-479. DOI: 10.1049/el:20063036.

Sarma A, Ratnam DV, Reddy DK. 2009. Modelling of low-latitude ionosphere using modified planar fit method for GAGAN. IET Radar Sonar Navig 3(6): 609-619. DOI: 10.1049/iet-rsn. 2009.0022.

Sarma AD, Sultana Q, Srinivas VS. 2010. Augmentation of Indian regional navigation satellite system to improve dilution of precision. J Navig Cambridge Univ Press 63(02): 313-321. DOI: $10.1017 / \mathrm{S} 037346330999035 \mathrm{X}$. 
Shi C, Gu S, Lou Y, Ge M. 2012. An improved approach to model ionospheric delays for single-frequency precise point positioning. Adv Space Res 49(12): 1698-1708. DOI: 10.1016/j. asr.2012.03.016.

Shukla AK, Das S, Shukla AP, Palsule VS. 2013. Approach for nearreal-time prediction of ionospheric delay using Klobuchar-like coefficients for Indian region. IET Radar Sonar Navig 7(1): 67-74. DOI: 10.1049/iet-rsn.2011.0371.

Srinivas VS, Sarma A, Achanta HK. 2016. Modeling of ionospheric time delay using anisotropic IDW with jackknife technique. IEEE Trans Geosci Remote Sens 54(1): 513-519. DOI: 10.1109/ TGRS.2015.2461017.

Sunehra D, Satyanarayana K, Viswanadh C, Sarma A. 2010. Estimation of total electron content and instrumental biases of low latitude global positioning system stations using Kalman filter. IETE J Res 56(5): 235-241. DOI: 10.4103/0377-2063.72772.
Venkata Ratnam D, Sarma A, Satya Srinivas V, Sreelatha P. 2011. Performance evaluation of selected ionospheric delay models during geomagnetic storm conditions in low-latitude region. Radio Sci 46(3), 1-6. DOI: 10.1029/2010RS004592.

Wang N, Li Z, Huo X, Li M, Yuan Y, Yuan C. 2019. Refinement of global ionospheric coefficients for GNSS applications: Methodology and results. Adv Space Res 63(1): 343-358. DOI: 10.1016/j. asr.2018.09.021.

Wang N, Yuan Y, Li Z, Huo X. 2016. Improvement of Klobuchar model for GNSS single-frequency ionospheric delay corrections. Adv Space Res 57(7): 1555-1569. DOI: 10.1016/j. asr.2016.01.010.

Zaminpardaz S, Teunissen PJ, Nadarajah N. 2017. IRNSS/NavIC single-point positioning: A service area precision analysis. Mar Geod 40(4): 259-274. DOI: 10.1080/01490419.2016.1269034.

Cite this article as: Desai MV \& Shah SN 2019. Estimation of ionospheric delay of NavIC/IRNSS signals using the Taylor Series Expansion. J. Space Weather Space Clim. 9, A23. 